\title{
On some identities in law involving exponential functionals of Brownian motion and Cauchy variable
}

\author{
Yuu Hariya*
}

\begin{abstract}
Let $B=\left\{B_{t}\right\}_{t \geq 0}$ be a one-dimensional standard Brownian motion, to which we associate the exponential additive functional $A_{t}=\int_{0}^{t} e^{2 B_{s}} d s, t \geq 0$. Starting from a simple observation of generalized inverse Gaussian distributions with particular sets of parameters, we show, with the help of a result by Matsumoto-Yor (2000), that for every $x \in \mathbb{R}$ and for every finite stopping time $\tau$ of the process $\left\{e^{-B_{t}} A_{t}\right\}_{t \geq 0}$, there holds the identity in law

$$
\begin{aligned}
& \left(e^{B_{\tau}} \sinh x+\beta\left(A_{\tau}\right), C e^{B_{\tau}} \cosh x+\hat{\beta}\left(A_{\tau}\right), e^{-B_{\tau}} A_{\tau}\right) \\
& \stackrel{(d)}{=}\left(\sinh \left(x+B_{\tau}\right), C \cosh \left(x+B_{\tau}\right), e^{-B_{\tau}} A_{\tau}\right),
\end{aligned}
$$

which extends an identity due to Bougerol (1983) in several aspects. Here $\beta=$ $\{\beta(t)\}_{t \geq 0}$ and $\hat{\beta}=\{\hat{\beta}(t)\}_{t \geq 0}$ are one-dimensional standard Brownian motions, $C$ is a standard Cauchy variable, and $B, \beta, \hat{\beta}$ and $C$ are independent. Using an argument relevant to derivation of the above identity, we also present some invariance formulae for Cauchy variable involving an independent Rademacher variable. $\llbracket \| \rrbracket$
\end{abstract}

\section{Introduction}

Let $B=\left\{B_{t}\right\}_{t \geq 0}$ be a one-dimensional Brownian motion starting from 0 and set

$$
A_{t}:=\int_{0}^{t} e^{2 B_{s}} d s, \quad t \geq 0
$$

This additive functional appears as the quadratic variation process of a geometric Brownian motion $e^{B_{t}}, t \geq 0$, and these exponential functionals of Brownian motion have importance in a number of fields such as option pricing in mathematical finance (see, e.g.,

${ }^{*}$ Mathematical Institute, Tohoku University, Aoba-ku, Sendai 980-8578, Japan.

E-mail: hariya@m.tohoku.ac.jp

Key Words and Phrases: Brownian motion; exponential functional; Bougerol's identity; Cauchy variable; generalized inverse Gaussian distribution.

2010 Mathematical Subject Classification: Primary 60J65; Secondary 60J55, 60E07. 
[11]), diffusion processes in random environments ([16] and [8] among others), stochastic analysis of Laplacians on hyperbolic spaces (see [18, Sections 7.4 and 7.5] and references therein) and so on. There have been extensive studies made on these functionals (see the monograph [31] by Yor and detailed surveys [23, 24] by Matsumoto and Yor) and various kinds of equalities and identities that give us deep understanding of their laws have been found, among which Bougerol's celebrated identity ([7]) states that for every fixed $t>0$,

$$
\beta\left(A_{t}\right) \stackrel{(d)}{=} \sinh B_{t}
$$

or more generally, for every fixed $t>0$ and $x \in \mathbb{R}$,

$$
e^{B_{t}} \sinh x+\beta\left(A_{t}\right) \stackrel{(d)}{=} \sinh \left(x+B_{t}\right)
$$

where $\beta=\{\beta(t)\}_{t \geq 0}$ is a Brownian motion independent of $B$; unless otherwise stated, any Brownian motion that appears in this paper is one-dimensional and standard, namely starting from 0 . The former identity (1.1) is particularly useful in deriving an explicit expression for the Mellin transform of the law of $A_{t}$.

To see that the latter holds, one may follow the inventive reasoning due to Alili and Dufresne for the case $x=0$ provided in [․, Appendix]. For a fixed $x \in \mathbb{R}$, we consider the process $Y^{x}=\left\{Y_{t}^{x}\right\}_{t \geq 0}$ given by

$$
Y_{t}^{x}=e^{-B_{t}} \sinh x+e^{-B_{t}} \int_{0}^{t} e^{B_{s}} d W_{s}, \quad t \geq 0,
$$

where $W=\left\{W_{t}\right\}_{t \geq 0}$ is a Brownian motion independent of $B$. By defining a Brownian motion $\beta^{x}=\left\{\beta_{t}^{x}\right\}_{t \geq 0}$ in such a way that

$$
\beta_{t}^{x}=\int_{0}^{t} \frac{-Y_{s}^{x} d B_{s}+d W_{s}}{\sqrt{1+\left(Y_{s}^{x}\right)^{2}}},
$$

Itô's formula entails that $Y^{x}$ satisfies the following stochastic differential equation (SDE):

$$
d Y_{t}^{x}=\sqrt{1+\left(Y_{t}^{x}\right)^{2}} d \beta_{t}^{x}+\frac{1}{2} Y_{t}^{x} d t, \quad Y_{0}^{x}=\sinh x,
$$

which is uniquely solved as

$$
Y_{t}^{x}=\sinh \left(x+\beta_{t}^{x}\right), \quad t \geq 0 .
$$

On the other hand, due to independence of $B$ and $W$, we may express $Y^{x}$ as

$$
Y_{t}^{x}=e^{-B_{t}} \sinh x+e^{-B_{t}} \beta\left(A_{t}\right), \quad t \geq 0,
$$


with $\beta=\{\beta(t)\}_{t \geq 0}$ another Brownian motion independent of $B$. Therefore for every fixed $t>0$,

$$
\begin{aligned}
Y_{t}^{x} & \stackrel{(d)}{=} e^{-B_{t}} \sinh x+\beta\left(e^{-2 B_{t}} A_{t}\right) \\
& \stackrel{(d)}{=} e^{B_{t}} \sinh x+\beta\left(A_{t}\right),
\end{aligned}
$$

where the first line is due to the scaling property of Brownian motion and the second follows from the identity in law

$$
\left(e^{-B_{t}}, e^{-2 B_{t}} A_{t}\right) \stackrel{(d)}{=}\left(e^{B_{t}}, A_{t}\right)
$$

thanks to the time reversal: $\left\{B_{t}-B_{t-s}\right\}_{0 \leq s \leq t} \stackrel{(d)}{=}\left\{B_{s}\right\}_{0 \leq s \leq t}$. Comparing (1.7) and (1.5) leads to (1.2). For further details as well as recent progress in the research on Bougerol's identity such as extensions to other processes, we refer the reader to the survey [27] by Vakeroudis; for a matrix-valued extension of the identity, see [2].

Following the notation in a series of papers [20, 21, 22, 24] by Matsumoto-Yor, we denote by $Z=\left\{Z_{t}\right\}_{t \geq 0}$ the process defined by

$$
Z_{t}:=e^{-B_{t}} A_{t}
$$

As studied in detail in [20, 21], this process is a diffusion process in its own natural filtration, which will be recalled in Remark 2.3 below. In the sequel we also denote by $C$ a standard Cauchy variable whose probability density is $\left\{\pi\left(1+x^{2}\right)\right\}^{-1}, x \in \mathbb{R}$. Given a real-valued process $X=\left\{X_{t}\right\}_{t \geq 0}$ and a point $a \in \mathbb{R}$, we denote by $\tau_{a}(X)$ the first hitting time of $X$ to $a$ :

$$
\tau_{a}(X):=\inf \left\{t \geq 0 ; X_{t}=a\right\}
$$

with convention that $\tau_{a}(X)=\infty$ when \{\}$=\emptyset$. One of the objectives of this paper is to show that Bougerol's identity may be extended in the following manner: in the statement below, three processes $\beta=\{\beta(t)\}_{t \geq 0}, \hat{\beta}=\{\hat{\beta}(t)\}_{t \geq 0}$ and $\hat{B}=\left\{\hat{B}_{t}\right\}_{t \geq 0}$ denote Brownian motions.

Theorem 1.1. Fix $x \in \mathbb{R}$. For any stopping time $\tau$ of the process $Z$ such that $0<\tau<$ $\infty$ a.s., we have

$$
\begin{aligned}
& \left(e^{B_{\tau}} \sinh x+\beta\left(A_{\tau}\right), C e^{B_{\tau}} \cosh x+\hat{\beta}\left(A_{\tau}\right), Z_{\tau}\right) \\
& \stackrel{(d)}{=}\left(\sinh \left(x+B_{\tau}\right), C \cosh \left(x+B_{\tau}\right), Z_{\tau}\right),
\end{aligned}
$$

or equivalently,

$$
\begin{aligned}
& \left(e^{B_{\tau}} \sinh x+\beta\left(A_{\tau}\right), \tau_{e^{B \tau} \cosh x}(\hat{B})+A_{\tau}, Z_{\tau}\right) \\
& \stackrel{(d)}{=}\left(\sinh \left(x+B_{\tau}\right), \tau_{\cosh \left(x+B_{\tau}\right)}(\hat{B}), Z_{\tau}\right),
\end{aligned}
$$


where in (1.9), B, $\beta, \hat{\beta}$ and $C$ (resp. $B$ and $C$ ) are independent on the left-(resp. right-)hand side while in (1.10), B, $\beta$ and $\hat{B}$ (resp. $B$ and $\hat{B}$ ) are independent on the left-(resp. right-)hand side.

Remark 1.1. (1) Once Theorem 1.1 is established, then its extension to the case where $\tau$ is only assumed to be finite a.s. is straightforward thanks to sample path continuity of $B, \beta$ and $\hat{\beta}$; indeed, if $\tau$ is such that $\tau<\infty$ a.s., then by Theorem 1.1, the identity (1.9) holds with $\tau$ replaced by $\max \{\tau, \delta\}$ for any $\delta>0$ and the above-mentioned extension follows by letting $\delta \rightarrow 0$. The same remark stands as to the identity (1.10) by noting the fact that $\tau_{a}(B) \stackrel{(d)}{=} a^{2} \tau_{1}(B)$ for any $a \in \mathbb{R}$ because of the scaling property of Brownian motion (see also (2.2) below).

(2) It should be noted that the natural filtration of the process $Z$ is strictly contained in that of the original Brownian motion $B$ (see [20, Theorem 1.6]); therefore it is true that $\tau$ in the statement of the theorem is a stopping time of $B$ but the converse is not true.

(3) It would be interesting to note that taking $x=0$ in (1.9), we have in particular

$$
\frac{\beta\left(A_{t}\right)}{Z_{t}} \stackrel{(d)}{=} \frac{\sinh B_{t}}{Z_{t}}
$$

for every fixed $t>0$, from which it follows that

$$
\beta\left(\frac{1}{A_{t}}\right) \stackrel{(d)}{=} \frac{1}{2}\left(\frac{e^{2 B_{t}}}{A_{t}}-\frac{1}{A_{t}}\right) .
$$

Indeed, thanks to the scaling property of Brownian motion and (1.8), the left-hand side of (1.11) is identical in law with

$$
\beta\left(\frac{e^{2 B_{t}}}{A_{t}^{2}} \cdot A_{t}\right) \stackrel{(d)}{=} \beta\left(\frac{1}{A_{t}}\right) .
$$

It is informative to mention that in view of (1.8), the right-hand side of (1.12) does give a symmetric random variable:

$$
-\left(\frac{e^{2 B_{t}}}{A_{t}}-\frac{1}{A_{t}}\right) \stackrel{(d)}{=} \frac{e^{2 B_{t}}}{A_{t}}-\frac{1}{A_{t}}
$$

Similarly to (1.12), it also holds that for every fixed $t>0$,

$$
\beta\left(e^{B_{t}}\right) \stackrel{(d)}{=} \frac{1}{2}\left(\sqrt{\frac{e^{3 B_{t}}}{A_{t}}}-\sqrt{\frac{e^{-B_{t}}}{A_{t}}}\right)
$$

if we consider $\left(1 / \sqrt{Z_{t}}\right) \sinh B_{t}$.

We also prove another extension of Bougerol's identity: given a real-valued process $X=\left\{X_{t}\right\}_{t \geq 0}$ and $\mu \in \mathbb{R}$, we denote $X^{(\mu)}=\left\{X_{t}^{(\mu)}:=X_{t}+\mu t\right\}_{t \geq 0}$. 
Theorem 1.2. Fix $x \in \mathbb{R}$. For any stopping time $\tau$ of the process $Z$ such that $0<\tau<$ $\infty$ a.s., it holds that

$$
\begin{aligned}
& \left(e^{B_{\tau}} \sinh x+\beta\left(A_{\tau}\right), e^{B_{\tau}}, Z_{\tau}\right) \\
& \stackrel{(d)}{=}\left(\sinh \left(x+B_{\tau}\right), \tau_{\cosh \left(x+B_{\tau}\right)}\left(\hat{B}^{\left(\cosh x / Z_{\tau}\right)}\right) / Z_{\tau}, Z_{\tau}\right),
\end{aligned}
$$

where both $\beta=\{\beta(t)\}_{t \geq 0}$ and $\hat{B}=\left\{\hat{B}_{t}\right\}_{t \geq 0}$ are Brownian motions independent of $B$.

In Alili-Gruet [1, it is shown that for every fixed $t>0$,

$$
\left(\beta\left(A_{t}\right), B_{t}\right) \stackrel{(d)}{=}\left((2 Y-1) \phi\left(B_{t}, \sqrt{R_{t}^{2}+B_{t}^{2}}\right), B_{t}\right),
$$

where on the right-hand side, $Y$ is an arcsine variable whose probability density function is $\{\pi \sqrt{x(1-x)}\}^{-1}, 0<x<1$, the function $\phi$ is defined by

$$
\phi(x, z)=\sqrt{2 e^{x}(\cosh z-\cosh x)}
$$

for two reals $x$ and $z$ fulfilling $z \geq|x|$, the process $R=\left\{R_{t}\right\}_{t \geq 0}$ is a two-dimensional Bessel process starting from 0 , and three elements $B, Y$ and $R$ are independent; for the identity (1.15) and its proof, we also refer to [19, Lemma 1]. In the case $x=0$, the identity (1.14) in the above theorem complements (1.15) in the sense that it keeps the expressions of first coordinates the same as in Bougerol's original identity (1.1).

Apart from the proof of Theorem 1.2 given in the next section, in order to convince the reader of the validity of (1.14), we recall the well-known fact that for every $a, \mu \in \mathbb{R}$ with $a \mu \geq 0$ and for every $\lambda \in \mathbb{R}$,

$$
\mathbb{E}\left[\exp \left\{-\frac{\lambda^{2}}{2} \tau_{a}\left(B^{(\mu)}\right)\right\}\right]=\exp \left\{-\left(\sqrt{\mu^{2}+\lambda^{2}}-\mu\right)|a|\right\}
$$

(see, e.g., [15, Exercise 3.5.10], [6, p. 301, Formula 2.0.1]), from which we easily deduce, by differentiating both sides with respect to $\lambda$, that when $\mu \neq 0$,

$$
\mathbb{E}\left[\tau_{a}\left(B^{(\mu)}\right)\right]=\frac{a}{\mu}
$$

Thanks to this formula, we see that for every $x \in \mathbb{R}$ and $t>0$,

$$
\begin{aligned}
\mathbb{E}\left[\tau_{\cosh \left(x+B_{t}\right)}\left(\hat{B}^{\left(\cosh x / Z_{t}\right)}\right) / Z_{t}\right] & =\frac{\mathbb{E}\left[\cosh \left(x+B_{t}\right)\right]}{\cosh x} \\
& =\frac{1}{2 \cosh x}\left\{e^{x} \mathbb{E}\left[e^{B_{t}}\right]+e^{-x} \mathbb{E}\left[e^{-B_{t}}\right]\right\}
\end{aligned}
$$

which, regardless of $x$, agrees with $\mathbb{E}\left[e^{B_{t}}\right]$ by symmetry of Brownian motion.

Proof of our Theorems 1.1 and 1.2 hinges upon the following simple observation: 
Proposition 1.1. Given $\mu>0$, let $f: \mathbb{R} \rightarrow \mathbb{R}$ be a measurable function such that

$$
\int_{\mathbb{R}} d y e^{-\mu \cosh y}|f(\sinh y)|<\infty .
$$

Then it holds that

$$
\int_{\mathbb{R}} d y e^{-\mu \cosh y} f(\sinh y)=\int_{0}^{\infty} \frac{d v}{v} \exp \left(-\frac{1}{2 v}\right) \exp \left(-\frac{\mu^{2}}{2} v\right) \mathbb{E}[f(\beta(v))] .
$$

Here $\beta=\{\beta(t)\}_{t \geq 0}$ is a Brownian motion.

Remark 1.2. Although not stated in an explicit manner in the existing literature, this is not the first time for the relation (1.17) to be noticed; see Remark 2.2 for more information.

We give a proof of the above proposition in the next section. The proposition also enables us to obtain some invariance formulae for Cauchy variable, which are of interest in their own right:

Theorem 1.3. Let $\varepsilon$ be a Rademacher (or symmetric Bernoulli) variable taking values \pm 1 with probability $1 / 2$ independently of the standard Cauchy variable $C$. It then holds that:

(i) for every $a \in \mathbb{R}$ with $|a| \geq 1$ and for every $\theta \in[-1,1]$,

$$
a C+\theta \sqrt{1+a^{2} C^{2}} \varepsilon \stackrel{(d)}{=}\left(a+\theta \sqrt{a^{2}-1} \varepsilon\right) C
$$

(ii) for every $a, \theta \in[-1,1]$,

$$
a C+\theta \sqrt{1+a^{2} C^{2}} \varepsilon \stackrel{(d)}{=} a C+\theta \sqrt{1-a^{2}} \varepsilon .
$$

As both sides of the claimed identities contain $a C$ in common, we refer to them as invariance formulae. In Subsection 3.1, we show that by applying these invariance formulae, identities in Theorem 1.1 are recovered partly. As for invariance of Cauchy variable, the following one would also be of interest:

$$
\frac{C e^{B_{\tau}} \cosh x+\hat{\beta}\left(A_{\tau}\right)}{\sqrt{1+\left(e^{B_{\tau}} \sinh x+\beta\left(A_{\tau}\right)\right)^{2}}} \stackrel{(d)}{=} C,
$$

which is an immediate consequence of the identity (1.9) in Theorem 1.1. Here on the left-hand side, four elements $B, C, \beta$ and $\hat{\beta}$ are independent, and $x$ is any real as well as $\tau$ is any finite stopping time of $Z$ (recall Remark 1.1(1)); notice that the random variable on the left-hand side of (1.18) is independent of $Z_{\tau}$ as (1.9) indicates. See also Remark 2.4(3) below. 
We give an outline of the paper. In Section 2, we give proofs of Proposition 1.1 and Theorems 1.1 1.3, we prove Proposition 1.1 and recall the definition of generalized inverse Gaussian (GIG for short) distributions in Subsection 2.1, in Subsection 2.2, we prove Theorems 1.1 and 1.2 preparing Propositions 2.2 and 2.3, the former of which is due to Matsumoto-Yor [20] and the latter of which concerns some properties of GIG laws relevant to the two theorems; in Subsection 2.3, we give a proof of Theorem 1.3 by utilizing a particular case of Lemma 2.1, whose assertion follows from Proposition 1.1 and is also used in the proof of Proposition 2.3. In Section 3, we provide some results related to, as well as deduced from, our discussions developed in Section 2. One of them is the derivation, in a self-contained way, of an identity in law due to Dufresne [9] that is another profound identity in the study of exponential functionals of Brownian motion. In Section 4, we give concluding remarks in relation to the SDE approach to the identity (1.2) introduced at the beginning of the present section. Finally in the appendix, we explore several facts relevant to the (unnormalized) density function of the so-called Hartman-Watson law, which appears in an explicit representation for the joint law of $e^{B_{t}}$ and $A_{t}$ given $t>0$, due to Yor [30].

Throughout the paper, all random variables as well as all stochastic processes are assumed to be defined on a common probability space whose probability measure is denoted by $\mathbb{P}$. Expectation relative to $\mathbb{P}$ is denoted by $\mathbb{E}$. We also suppose that the probability space we work in is nice enough to support regular conditional probabilities which we denote by the symbol $\mathbb{P}(\mid)$. We say that a random variable $X$ is symmetric if $-X$ has the same law as $X:-X \stackrel{(d)}{=} X$. Other notation and terminology will be introduced as needed.

\section{Proofs}

We devote this section to proofs of the results introduced in the previous section.

\subsection{Proof of Proposition 1.1}

In this subsection, we prove Proposition 1.1 and restate it in terms of random variables, to which we relate the notion of generalized inverse Gaussian distributions. We recall that for every real $a \neq 0$, the law of $\tau_{a}(B)$ is given by

$$
\mathbb{P}\left(\tau_{a}(B) \in d v\right)=\frac{|a|}{\sqrt{2 \pi v^{3}}} \exp \left(-\frac{a^{2}}{2 v}\right) d v, \quad v>0,
$$

which may be seen from (1.16) as well as from the fact that by reflection principle,

$$
\tau_{a}(B) \stackrel{(d)}{=} \frac{a^{2}}{B_{1}^{2}} .
$$


In the sequel we denote by

$$
\operatorname{Argsh} x \equiv \log \left(x+\sqrt{1+x^{2}}\right), \quad x \in \mathbb{R},
$$

the inverse function of the hyperbolic sine function.

Proof of Proposition 1.1. By (1.16) and (2.1), we may write

$$
\begin{aligned}
e^{-\mu \cosh y} & =\mathbb{E}\left[e^{-\frac{\mu^{2}}{2} \tau_{\cosh y}(B)}\right] \\
& =\int_{0}^{\infty} d v \frac{\cosh y}{\sqrt{2 \pi v^{3}}} \exp \left(-\frac{\cosh ^{2} y}{2 v}\right) \exp \left(-\frac{\mu^{2}}{2} v\right)
\end{aligned}
$$

for every $\mu>0$ and $y \in \mathbb{R}$. Plugging the last expression into the left-hand side of (1.17) and using Fubini's theorem, we have the equality

$$
\begin{aligned}
& \int_{\mathbb{R}} d y e^{-\mu \cosh y} f(\sinh y) \\
& =\int_{0}^{\infty} \frac{d v}{v} \exp \left(-\frac{1}{2 v}\right) \exp \left(-\frac{\mu^{2}}{2} v\right) \int_{\mathbb{R}} d y \frac{\cosh y}{\sqrt{2 \pi v}} \exp \left(-\frac{\sinh ^{2} y}{2 v}\right) f(\sinh y) .
\end{aligned}
$$

Changing the variables with $y=\operatorname{Argsh} x, x \in \mathbb{R}$, in the integral with respect to $y$, leads to the desired expression.

It is possible to rephrase (1.17) as an identity in law. For every $\mu>0$, we consider a random variable $z_{\mu}$ whose law is given by

$$
\mathbb{P}\left(z_{\mu} \in d x\right)=\frac{1}{2 K_{0}(\mu)} e^{-\mu \cosh x} d x, \quad x \in \mathbb{R} .
$$

Here and in what follows, for every $\nu \in \mathbb{R}$, we denote by $K_{\nu}$ the modified Bessel function of the third kind (Macdonald function) of order $\nu$, one of whose integral representations is given by

$$
K_{\nu}(z)=\frac{1}{2} \int_{\mathbb{R}} d x e^{-z \cosh x-\nu x}=\frac{1}{2} \int_{\mathbb{R}} d x e^{-z \cosh x} \cosh (\nu x), \quad z>0
$$

(cf. [17, Equation (5.10.23)]). The statement of Proposition 1.1 is then rephrased as

$$
\sinh z_{\mu} \stackrel{(d)}{=} \beta\left(\frac{e^{z_{\mu}}}{\mu}\right)
$$

for every $\mu>0$, where on the right-hand side, $\beta=\{\beta(t)\}_{t \geq 0}$ is a Brownian motion independent of $z_{\mu}$; indeed, we divide by $2 K_{0}(\mu)$ both sides of (1.17) and change the variables with $v=e^{y} / \mu, y \in \mathbb{R}$, on the right-hand side to see that

$$
\begin{aligned}
\mathbb{E}\left[f\left(\sinh z_{\mu}\right)\right] & =\frac{1}{2 K_{0}(\mu)} \int_{\mathbb{R}} d y e^{-\mu \cosh y} \mathbb{E}\left[f\left(\beta\left(e^{y} / \mu\right)\right)\right] \\
& =\mathbb{E}\left[f\left(\beta\left(e^{z_{\mu}} / \mu\right)\right)\right]
\end{aligned}
$$


for any bounded measurable function $f$ on $\mathbb{R}$. As will be seen in the next subsection, if we let $\mu$ vary according to the law of $1 / Z_{t} \equiv e^{B_{t}} / A_{t}$ for a fixed $t>0$, or in other words, if we integrate both sides of (2.6) $)$ over $\mu>0$ with respect to $\mathbb{P}\left(e^{B_{t}} / A_{t} \in d \mu\right)$, then Bougerol's identity (1.1) is recovered.

We may relate the random variable $z_{\mu}$ to a generalized inverse Gaussian (GIG) law: recall from [21, Section 9] that given three parameters $\nu \in \mathbb{R}$ and $a, b>0$, a random variable $I \equiv I_{a, b}^{(\nu)}$ is said to follow the $\operatorname{GIG}(\nu ; a, b)$ distribution if

$$
P(I \in d v)=\left(\frac{b}{a}\right)^{\nu} \frac{v^{\nu-1}}{2 K_{\nu}(a b)} \exp \left\{-\frac{1}{2}\left(\frac{a^{2}}{v}+b^{2} v\right)\right\} d v, \quad v>0 .
$$

To verify that the right-hand side does give a probability distribution, it is meaningful to recall another integral representation of $K_{\nu}$, which one obtains from (2.4) by changing the variables with $e^{-x}=2 v / z, v>0$ :

$$
K_{\nu}(z)=\frac{1}{2}\left(\frac{z}{2}\right)^{-\nu} \int_{0}^{\infty} d v v^{\nu-1} \exp \left(-v-\frac{z^{2}}{4 v}\right), \quad z>0
$$

(see e.g., [17, Equation (5.10.25)]); a probabilistic expression for (2.8) in terms of gamma variable, will be given in (3.8). In a similar way to (2.6) , we see that $e^{z_{\mu}} / \mu$ is $\operatorname{GIG}(0 ; 1, \mu)$ distributed:

$$
\frac{e^{z_{\mu}}}{\mu} \stackrel{(d)}{=} I_{1, \mu}^{(0)}, \quad \text { or equivalently, } e^{z_{\mu}} \stackrel{(d)}{=} I_{\sqrt{\mu}, \sqrt{\mu}}^{(0)}
$$

Indeed, for any bounded measurable function $f$ on $(0, \infty)$, we have

$$
\begin{aligned}
\mathbb{E}\left[f\left(e^{z_{\mu}} / \mu\right)\right] & =\frac{1}{2 K_{0}(\mu)} \int_{\mathbb{R}} d x e^{-\mu \cosh x} f\left(e^{x} / \mu\right) \\
& =\frac{1}{2 K_{0}(\mu)} \int_{0}^{\infty} \frac{d v}{v} \exp \left(-\frac{1}{2 v}\right) \exp \left(-\frac{\mu^{2}}{2} v\right) f(v) \\
& =\mathbb{E}\left[f\left(I_{1, \mu}^{(0)}\right)\right]
\end{aligned}
$$

by (2.7), which shows the former identity in (2.9). Here for the second line, we changed the variables with $e^{x} / \mu=v$. The latter follows from the elementary property that $c^{2} I_{a, b}^{(\nu)} \stackrel{(d)}{=} I_{a c, a / c}^{(\nu)}$ for every $\nu \in \mathbb{R}$ and $a, b, c>0$. The above computation also reveals the following representation of $K_{0}$, which is immediate from (2.8) as well, and will be recalled repeatedly:

$$
K_{0}(z)=\frac{1}{2} \int_{0}^{\infty} \frac{d v}{v} \exp \left(-\frac{1}{2 v}\right) \exp \left(-\frac{z^{2}}{2} v\right), \quad z>0 .
$$

In the case $\nu=0$, the GIG distribution is also referred to as Halphen's harmonic law or the hyperbola distribution; see [14] and [26] (be aware that the above parametrization for GIG laws is slightly different from the one used in these references). We also remark 
that when $a, \mu>0$, the stopping time $\tau_{a}\left(B^{(\mu)}\right)$ as appeared in (1.16) follows the inverse Gaussian distribution, namely it is identical in law with $I_{a, \mu}^{(-1 / 2)}$.

For later use in Remark 2.4, it is convenient to observe here that the following convergence in law takes place:

Proposition 2.1. $I_{\sqrt{\mu}, \sqrt{\mu}}^{(0)}$ converges in law to 1 as $\mu \rightarrow \infty$. The same convergence holds true for $e^{z_{\mu}}$ by virtue of (2.9).

Proof. Let $f:(0, \infty) \rightarrow \mathbb{R}$ be bounded and continuous and note the equalities

$$
\begin{aligned}
& \mathbb{E}\left[f\left(I_{\sqrt{\mu}, \sqrt{\mu}}^{(0)}\right)\right] \times \sqrt{\frac{2 \mu}{\pi}} e^{\mu} K_{0}(\mu) \\
& =\sqrt{\frac{\mu}{2 \pi}} \int_{0}^{\infty} \frac{d v}{v} \exp \left\{-\frac{\mu}{2}\left(\sqrt{v}-\frac{1}{\sqrt{v}}\right)^{2}\right\} f(v) \\
& =\int_{\mathbb{R}} \frac{d x}{\sqrt{2 \pi}} \exp \left(-\frac{x^{2}}{2}\right) \frac{1}{\sqrt{1+x^{2} /(4 \mu)}} f\left(\left(\frac{x}{2 \sqrt{\mu}}+\sqrt{1+\frac{x^{2}}{4 \mu}}\right)^{2}\right),
\end{aligned}
$$

where we changed the variables with $\sqrt{v}-1 / \sqrt{v}=x / \sqrt{\mu}, x \in \mathbb{R}$, for the last line. We let $\mu \rightarrow \infty$ : it is known that for any $\nu \in \mathbb{R}$,

$$
\sqrt{\frac{2 \mu}{\pi}} e^{\mu} K_{\nu}(\mu) \rightarrow 1
$$

(see [17, Equation (5.16.5)]); on the other hand, thanks to boundedness and continuity of $f$, the bounded convergence theorem entails that the last expression of the above equalities converges to

$$
\int_{\mathbb{R}} \frac{d x}{\sqrt{2 \pi}} \exp \left(-\frac{x^{2}}{2}\right) \times f(1)=f(1)
$$

Therefore we have

$$
\lim _{\mu \rightarrow \infty} \mathbb{E}\left[f\left(I_{\sqrt{\mu}, \sqrt{\mu}}^{(0)}\right)\right]=f(1)
$$

for any bounded and continuous $f$ on $(0, \infty)$, which shows the proposition.

The above proposition may also be deduced from Equation (3.10) or (3.11) in [14]; see Remark 2.4(2) below as to those two equations.

\subsection{Proof of Theorems 1.1 and 1.2}

In this subsection we prove Theorems 1.1 and 1.2. We begin with a proposition, the assertion of which is a particular case of that of [20, Proposition 1.7] as we are dealing with the Brownian motion without drift. 
Proposition 2.2. Let $\tau$ be a stopping time of the process $Z$ such that $0<\tau<\infty$ a.s. and $\mu$ a positive real. Then the conditional distribution of $e^{B_{\tau}} / \mu$ given $1 / Z_{\tau}=\mu$ coincides with the $\operatorname{GIG}(0 ; 1, \mu)$ distribution; in other words, conditionally on $1 / Z_{\tau}=\mu$, it holds that

$$
B_{\tau} \stackrel{(d)}{=} z_{\mu}
$$

in view of (2.9).

Remark 2.1. Since $z_{\mu}$ is symmetric for each $\mu>0$ as seen from (2.3), the relation (2.12) indicates that $B_{\tau}$ is symmetric, which fact may be regarded as a reflection of strict containedness of the natural filtration of $Z$ in that of $B$ referred to in Remark 1.1(2).

To keep the paper self-contained as much as possible, we provide in Remark 2.3 below reasoning to deduce Proposition 2.2 from the diffusion property of $Z$ discussed in detail in [20, 21]. Thanks to the proposition, Theorems 1.1 and 1.2 are immediate consequences of the following extensions of the identity (2.5): as in the statements of those two theorems, we let $\beta=\{\beta(t)\}_{t \geq 0}, \hat{\beta}=\{\hat{\beta}(t)\}_{t \geq 0}$ and $\hat{B}=\left\{\hat{B}_{t}\right\}_{t \geq 0}$ denote Brownian motions.

Proposition 2.3. For every fixed $\mu>0$ and $x \in \mathbb{R}$, we have the following identities in law:

(i) it holds that

$$
\begin{aligned}
& \left(e^{z_{\mu}} \sinh x+\beta\left(e^{z_{\mu}} / \mu\right), C e^{z_{\mu}} \cosh x+\hat{\beta}\left(e^{z_{\mu}} / \mu\right)\right) \\
& \stackrel{(d)}{=}\left(\sinh \left(x+z_{\mu}\right), C \cosh \left(x+z_{\mu}\right)\right),
\end{aligned}
$$

or equivalently,

$$
\begin{aligned}
& \left(e^{z_{\mu}} \sinh x+\beta\left(e^{z_{\mu}} / \mu\right), \tau_{e^{z_{\mu}} \cosh x}(\hat{B})+e^{z_{\mu}} / \mu\right) \\
& \stackrel{(d)}{=}\left(\sinh \left(x+z_{\mu}\right), \tau_{\cosh \left(x+z_{\mu}\right)}(\hat{B})\right),
\end{aligned}
$$

where in (2.13) $, z_{\mu}, \beta, \hat{\beta}$ and $C$ (resp. $z_{\mu}$ and $C$ ) are independent on the left-(resp. right-)hand side while in (2.14) $, z_{\mu}, \beta$ and $\hat{B}$ (resp. $z_{\mu}$ and $\hat{B}$ ) are independent on the left-(resp. right-)hand side;

(ii) it holds that

$$
\begin{aligned}
& \left(e^{z_{\mu}} \sinh x+\beta\left(e^{z_{\mu}} / \mu\right), e^{z_{\mu}}\right) \\
& \stackrel{(d)}{=}\left(\sinh \left(x+z_{\mu}\right), \mu \tau_{\cosh \left(x+z_{\mu}\right)}\left(\hat{B}^{(\mu \cosh x)}\right)\right),
\end{aligned}
$$

where $\beta$ and $\hat{B}$ are independent of $z_{\mu}$. 
Proof of Theorems 1.1 and 1.2. Assertions of Theorems 1.1 and 1.2 follow immediately from (i) and (ii) of Proposition 2.3, respectively, since it holds that by Proposition 2.2.

$$
\int_{0}^{\infty} \mathbb{P}\left(1 / Z_{\tau} \in d \mu\right) \mathbb{E}\left[f\left(z_{\mu}, e^{z_{\mu}} / \mu, 1 / \mu\right)\right]=\mathbb{E}\left[f\left(B_{\tau}, A_{\tau}, Z_{\tau}\right)\right]
$$

for any bounded measurable function $f: \mathbb{R} \times(0, \infty)^{2} \rightarrow \mathbb{R}$.

Remark 2.2. If we denote by $N$ a one-dimensional standard Gaussian random variable independent of $I_{1, \mu}^{(0)}$, then by (2.9), the first coordinate in the left-hand side of (2.13) is identical in law with

$$
\mu \sinh x \cdot I_{1, \mu}^{(0)}+\sqrt{I_{1, \mu}^{(0)}} N
$$

If we replace $I_{1, \mu}^{(0)}$ by a generic nonnegative random variable $X$ and $\mu \sinh x$ by a generic constant, then by adding an additional constant, the operation as in (2.16) of producing a new probability distribution is often called the normal mean-variance mixture with mixing law $X$ in the literature. For instance, in the final section of [3], BarndorffNielsen uses $X=I_{a, b}^{(\nu)}$ as the mixing law to introduce briefly what is referred to as the generalized hyperbolic distribution; see also [14] and [26]. Although it is not stated in an explicit form as the identity between first coordinates in (2.13), a connection between the generalized hyperbolic and hyperbola distributions is discussed later by BarndorffNielsen [4, Section 5]. On the other hand, the identity between second coordinates in (2.13), as well as in (2.14), and that in (2.15) seem to be new to our knowledge; in particular, the latter identity reveals a connection between the hyperbola and inverse Gaussian distributions. Moreover, the identity (2.15) enables us to obtain a certain relationship among the laws of $\sinh \left(x+z_{\mu}\right)$ for different values of $x$; see Remark 2.4(4). As for the notions of the hyperbola and inverse Gaussian distributions, recall their description given just before Proposition 2.1.

Before providing a proof of Proposition 2.3, we explain how to deduce Proposition 2.2 , and provide related facts.

Remark 2.3. (1) First observe that $\lim _{t \rightarrow \infty} A_{t}=\infty$ a.s.; indeed, by Lamperti's well-known relation (see, e.g., [25, Chapter XI, Exercise (1.28)]), there exists a two-dimensional Bessel process $R=\{R(t)\}_{t \geq 0}$ starting from 1, such that

$$
e^{B_{t}}=R\left(A_{t}\right), \quad t \geq 0
$$

from which absurdity of $\mathbb{P}\left(\lim _{t \rightarrow \infty} A_{t}<\infty\right)>0$ follows since this positivity should imply existence of $\lim _{t \rightarrow \infty} B_{t}$ with positive probability due to sample path continuity of $R$. Therefore we have the relation

$$
\int_{t}^{\infty} \frac{d s}{Z_{s}^{2}}=\left[-\frac{1}{A_{s}}\right]_{t}^{\infty}=\frac{1}{A_{t}}
$$


for all $t>0$ a.s., and hence

$$
e^{B_{t}}=\left(Z_{t} \int_{t}^{\infty} \frac{d s}{Z_{s}^{2}}\right)^{-1}, \quad t>0, \text { a.s. }
$$

The former relation (2.17) reveals that the process $Z$ is transient: $\lim _{t \rightarrow \infty} Z_{t}=\infty$ a.s. We also recall from [20, Theorem 1.6] that $Z$ is a diffusion process in its natural filtration with the infinitesimal generator

$$
\frac{1}{2} z^{2} \frac{d^{2}}{d z^{2}}+\left\{\frac{1}{2} z+\frac{K_{1}}{K_{0}}\left(\frac{1}{z}\right)\right\} \frac{d}{d z}
$$

If we denote by $\left(Z=\left\{Z_{t}\right\}_{t \geq 0},\left\{P_{z}\right\}_{z \geq 0}\right)$ the strong Markov family associated with (2.19) so that $P_{z}\left(Z_{0}=z\right)=1$, then Proposition 1.7 of [20] referred to at the beginning of this subsection tells us that for every $z>0$, the functional

$$
\int_{0}^{\infty} \frac{d s}{Z_{s}^{2}}
$$

is distributed, under the probability measure $P_{z}$, as $1 / I_{1,1 / z}^{(0)}$. Combining this fact with the expression (2.18) of geometric Brownian motion in terms of $Z$, leads to the statement of Proposition 2.2 in such a way that for $\mu>0$ and $u>0$,

$$
\begin{aligned}
\mathbb{P}\left(e^{B_{\tau}} \in d u \mid Z_{\tau}=1 / \mu\right) & =P_{1 / \mu}\left(\mu\left(\int_{0}^{\infty} \frac{d s}{Z_{s}^{2}}\right)^{-1} \in d u\right) \\
& =\mathbb{P}\left(\mu I_{1, \mu}^{(0)} \in d u\right) .
\end{aligned}
$$

(2) It would also be worthwhile mentioning that given $\mu>0$, by considering the process $\left\{1 / Z_{t}\right\}_{t \geq 0}$ under $P_{1 / \mu}$, the functional (2.20) is seen to be identical in law with the first hitting time to 0 by a diffusion process starting from $\mu$ whose infinitesimal generator is given by

$$
\frac{1}{2} \frac{d^{2}}{d z^{2}}+\left\{\frac{1}{2 z}-\frac{K_{1}}{K_{0}}(z)\right\} \frac{d}{d z}
$$

A direct computation shows that for each fixed $\lambda \in \mathbb{R}$, the function

$$
E_{1 / \mu}\left[\exp \left(-\frac{\lambda^{2}}{2} \int_{0}^{\infty} \frac{d s}{Z_{s}^{2}}\right)\right]=\frac{K_{0}\left(\mu \sqrt{1+\lambda^{2}}\right)}{K_{0}(\mu)}, \quad \mu>0
$$

solves the eigenvalue problem

$$
f^{\prime \prime}(\mu)+\left\{\frac{1}{\mu}-2 \frac{K_{1}}{K_{0}}(\mu)\right\} f^{\prime}(\mu)=\lambda^{2} f(\mu)
$$

associated with the above second-order differential operator. We refer to a more general fact by Barndorff-Nielsen et al. [5] that any $\operatorname{GIG}(\nu ; a, b)$ distribution with nonpositive 
$\nu$ is realized as the law of a first hitting time of some diffusion process. The above representation (2.21) follows readily from (2.10).

(3) We recall from [30, Proposition 2] (see also [31, p. 43]) that whenever $t>0$, the joint law of $e^{B_{t}}$ and $A_{t}$ is given by

$$
\mathbb{P}\left(e^{B_{t}} \in d u, A_{t} \in d v\right)=\frac{1}{u v} \exp \left(-\frac{1+u^{2}}{2 v}\right) \Theta_{u / v}(t) d u d v, \quad u, v>0,
$$

where for every $r>0$, the function $\Theta_{r}(t), t>0$, is an unnormalized density of the so-called Hartman-Watson law with parameter $r$, which is characterized by

$$
\int_{0}^{\infty} d t e^{-\lambda^{2} t / 2} \Theta_{r}(t)=I_{|\lambda|}(r), \quad \lambda \in \mathbb{R} .
$$

Here for every $\nu \in \mathbb{R}$, the function $I_{\nu}$ denotes the modified Bessel function of the first kind of order $\nu$; see [17, Section 5.7] for definition. As for the formula (2.22), we also refer to [23, Theorem 4.1] as well as [18, Theorem 7.5.1] for its different proof than the original one, based on the Sturm-Liouville theory. The following explicit representation for $\Theta_{r}(t)$ is shown in [29]:

$$
\Theta_{r}(t)=\frac{r}{\sqrt{2 \pi^{3} t}} \int_{0}^{\infty} d y \exp \left(\frac{\pi^{2}-y^{2}}{2 t}\right) \exp (-r \cosh y) \sinh y \sin \left(\frac{\pi y}{t}\right) .
$$

Owing to (2.22), we find that

$$
\mathbb{P}\left(A_{t} \in d v, 1 / Z_{t} \in d \mu\right)=\frac{1}{\mu} \Theta_{\mu}(t) \frac{1}{v} \exp \left(-\frac{1}{2 v}\right) \exp \left(-\frac{\mu^{2}}{2} v\right) d v d \mu, \quad v, \mu>0,
$$

and that in particular,

$$
\mathbb{P}\left(1 / Z_{t} \in d \mu\right)=\frac{2}{\mu} K_{0}(\mu) \Theta_{\mu}(t) d \mu, \quad \mu>0 .
$$

Indeed, for every $s>0$ and $\mu>0$,

$$
\begin{aligned}
\mathbb{P}\left(A_{t} \leq s, 1 / Z_{t} \leq \mu\right) & =\int_{0}^{s} \frac{d v}{v} \int_{0}^{\mu v} \frac{d u}{u} \exp \left(-\frac{1+u^{2}}{2 v}\right) \Theta_{u / v}(t) \\
& =\int_{0}^{\mu} \frac{d u}{u} \Theta_{u}(t) \int_{0}^{s} \frac{d v}{v} \exp \left(-\frac{1}{2 v}\right) \exp \left(-\frac{u^{2}}{2} v\right)
\end{aligned}
$$

and letting $s \rightarrow \infty$ yields

$$
\mathbb{P}\left(1 / Z_{t} \leq \mu\right)=2 \int_{0}^{\mu} \frac{d u}{u} \Theta_{u}(t) K_{0}(u)
$$

thanks to (2.10), where for the second line in (2.27), we changed the variable $u$ into $v u$ and used Fubini's theorem. From (2.25) and (2.26) , we see that

$$
\mathbb{P}\left(A_{t} \in d v \mid 1 / Z_{t}=\mu\right)=\frac{1}{2 K_{0}(\mu)} \frac{1}{v} \exp \left(-\frac{1}{2 v}\right) \exp \left(-\frac{\mu^{2}}{2} v\right) d v, \quad v>0, \mu>0,
$$

which is nothing but the case $\tau=t$ in Proposition 2.2 because conditionally on $1 / Z_{t}=\mu$, we have the expression $A_{t}=e^{B_{t}} / \mu$. 
We proceed to the proof of Proposition 2.3. In what follows the symbol $i$ stands for $\sqrt{-1}$.

Lemma 2.1. For every $\mu>0, \lambda \geq 0$ and $\xi \in \mathbb{R}$, it holds that for all $x \in \mathbb{R}$,

$$
\begin{aligned}
& \int_{\mathbb{R}} d y e^{-\mu \cosh y} e^{-\lambda \cosh (x+y)} e^{i \xi \sinh (x+y)} \\
& =\int_{0}^{\infty} \frac{d v}{v} \exp \left(-\frac{1}{2 v}\right) \exp \left(-\frac{\mu^{2}+\lambda^{2}+\xi^{2}}{2} v\right) e^{-\mu \lambda v \cosh x} e^{i \mu \xi v \sinh x} .
\end{aligned}
$$

Proof. By translation, the left-hand side of (2.28) is equal to

$$
\int_{\mathbb{R}} d y e^{-\mu \cosh (y-x)} e^{-\lambda \cosh y} e^{i \xi \sinh y}
$$

which is rewritten, by the relation $\cosh (y-x)=\cosh y \cosh x-\sinh y \sinh x$, as

$$
\int_{\mathbb{R}} d y e^{-(\mu \cosh x+\lambda) \cosh y} e^{\mu \sinh x \sinh y} e^{i \xi \sinh y} .
$$

Applying Proposition 1.1 with exponent $\mu$ therein replaced by $\mu \cosh x+\lambda$, we see that the last expression is further rewritten as

$$
\int_{0}^{\infty} \frac{d v}{v} \exp \left(-\frac{1}{2 v}\right) \exp \left\{-\frac{(\mu \cosh x+\lambda)^{2}}{2} v\right\} \mathbb{E}[\exp \{(\mu \sinh x+i \xi) \beta(v)\}] .
$$

As the expectation in this integral is equal to

$$
\exp \left\{\frac{(\mu \sinh x+i \xi)^{2}}{2} v\right\}=\exp \left(\frac{\mu^{2} \sinh ^{2} x-\xi^{2}}{2} v\right) e^{i \mu \xi v \sinh x}
$$

rearranging terms in the integral leads to the right-hand side of $(2.28)$ and ends the proof.

We are in a position to prove Proposition 2.3; as the proof shows, the assertion (i) is a rewriting of $(2.28)$ in terms of random variables.

Proof of Proposition 2.3. First we prove (i) using Lemma 2.1 prepared above. To this end, we replace $\lambda \geq 0$ in (2.28) by $|\lambda|$ for an arbitrary $\lambda \in \mathbb{R}$. In view of (2.3), we may rewrite the left-hand side of (2.28) as

$$
\begin{aligned}
& 2 K_{0}(\mu) \mathbb{E}\left[\exp \left\{-|\lambda| \cosh \left(x+z_{\mu}\right)\right\} \exp \left\{i \xi \sinh \left(x+z_{\mu}\right)\right\}\right] \\
& =2 K_{0}(\mu) \mathbb{E}\left[\exp \left\{i \lambda C \cosh \left(x+z_{\mu}\right)\right\} \exp \left\{i \xi \sinh \left(x+z_{\mu}\right)\right\}\right] .
\end{aligned}
$$

Here $C$ is independent of $z_{\mu}$ and we have used the fact that $\mathbb{E}[\exp (i \alpha C)]=\exp (-|\alpha|)$ for any $\alpha \in \mathbb{R}$. On the other hand, by changing the variables with $v=e^{y} / \mu, y \in \mathbb{R}$, the right-hand side of (2.28) with $\lambda$ replaced by $|\lambda|$, is rewritten as

$$
\begin{aligned}
& \int_{\mathbb{R}} d y e^{-\mu \cosh y} \times \exp \left(-\frac{\lambda^{2}}{2} \frac{e^{y}}{\mu}-|\lambda| e^{y} \cosh x\right) \times \exp \left(-\frac{\xi^{2}}{2} \frac{e^{y}}{\mu}+i \xi e^{y} \sinh x\right) \\
& =: \int_{\mathbb{R}} d y e^{-\mu \cosh y} \times I \times I I, \text { say. }
\end{aligned}
$$


Denoting by $\beta=\{\beta(t)\}_{t \geq 0}$ a Brownian motion independent of $C$, we have

$$
I=\mathbb{E}\left[\exp \left\{i \lambda\left(C e^{y} \cosh x+\beta\left(e^{y} / \mu\right)\right)\right\}\right]
$$

and likewise

$$
I I=\mathbb{E}\left[\exp \left\{i \xi\left(e^{y} \sinh x+\beta\left(e^{y} / \mu\right)\right)\right\}\right]
$$

Plugging these into (2.30) and noting (2.3) again, we conclude that the righ-hand side of (2.28) admits the expression

$$
2 K_{0}(\mu) \mathbb{E}\left[\exp \left\{i \lambda\left(C e^{z_{\mu}} \cosh x+\hat{\beta}\left(e^{z_{\mu}} / \mu\right)\right)\right\} \exp \left\{i \xi\left(e^{z_{\mu}} \sinh x+\beta\left(e^{z_{\mu}} / \mu\right)\right)\right\}\right],
$$

where $\hat{\beta}=\{\hat{\beta}(t)\}_{t \geq 0}$ is another Brownian motion, and $z_{\mu}, \beta, \hat{\beta}$ and $C$ are independent. Since the last expression agrees with (2.29) for any $\lambda, \xi \in \mathbb{R}$, we obtain the identity (2.13) by successive use of the injectivity of Fourier transform.

To prove the equivalent expression (2.14), it suffices to note the fact that for two independent Brownian motions $\beta$ and $\hat{B}=\left\{\hat{B}_{t}\right\}_{t \geq 0}$, there holds the identity in law $\beta\left(\tau_{a}(\hat{B})\right) \stackrel{(d)}{=} a C$ for every $a \in \mathbb{R}$ :

$$
\begin{aligned}
\mathbb{E}\left[\exp \left\{i \xi \beta\left(\tau_{a}(\hat{B})\right)\right\}\right] & =\mathbb{E}\left[\exp \left\{-\frac{\xi^{2}}{2} \tau_{a}(\hat{B})\right\}\right] \\
& =\exp (-|\xi||a|),
\end{aligned}
$$

where $\xi \in \mathbb{R}$ is arbitrary and the second line is due to (1.16). Using this well-known fact, we may rephrase the identity $(\underline{2.13})$ as

$$
\left(e^{z_{\mu}} \sinh x+\beta\left(e^{z_{\mu}} / \mu\right), \hat{\beta}\left(\tau_{e^{z \mu} \cosh x}(\hat{B})+e^{z_{\mu}} / \mu\right)\right) \stackrel{(d)}{=}\left(\sinh \left(x+z_{\mu}\right), \hat{\beta}\left(\tau_{\cosh \left(x+z_{\mu}\right)}(\hat{B})\right)\right)
$$

where $\hat{\beta}=\{\hat{\beta}(t)\}_{t \geq 0}$ is a Brownian motion independent of $z_{\mu}, \beta$ and $\hat{B}$. Taking the joint Fourier transform on both sides leads to coincidence of joint Fourier-Laplace transforms in such a way that for any $\lambda, \xi \in \mathbb{R}$,

$$
\begin{aligned}
& \mathbb{E}\left[\exp \left\{i \lambda\left(e^{z_{\mu}} \sinh x+\beta\left(e^{z_{\mu}} / \mu\right)\right)\right\} \exp \left\{-\frac{\xi^{2}}{2}\left(\tau_{e^{z_{\mu}} \cosh x}(\hat{B})+\frac{e^{z_{\mu}}}{\mu}\right)\right\}\right] \\
& =\mathbb{E}\left[\exp \left\{i \lambda \sinh \left(x+z_{\mu}\right)\right\} \exp \left\{-\frac{\xi^{2}}{2} \tau_{\cosh \left(x+z_{\mu}\right)}(\hat{B})\right\}\right]
\end{aligned}
$$

which proves the identity (2.14) owing to the injectivity of Fourier and Laplace transforms. From the above argument it is obvious that (2.14) implies (2.13) conversely. 
We turn to the proof of (ii). Let $f: \mathbb{R} \rightarrow \mathbb{R}$ be bounded and measurable, and take $\lambda \in \mathbb{R}$ arbitrarily. For a Brownian motion $\beta=\{\beta(t)\}_{t \geq 0}$ independent of $z_{\mu}$, we compute

$$
\begin{aligned}
& 2 K_{0}(\mu) \mathbb{E}\left[f\left(e^{z_{\mu}} \sinh x+\beta\left(e^{z_{\mu}} / \mu\right)\right) \exp \left(-\frac{\lambda^{2}}{2} \frac{e^{z_{\mu}}}{\mu}\right)\right] \\
& =\int_{\mathbb{R}} d y e^{-\mu \cosh y} \exp \left(-\frac{\lambda^{2}}{2} \frac{e^{y}}{\mu}\right) \mathbb{E}\left[f\left(e^{y} \sinh x+\beta\left(e^{y} / \mu\right)\right)\right] \\
& =\int_{0}^{\infty} \frac{d v}{v} \exp \left(-\frac{1}{2 v}\right) \exp \left(-\frac{\mu^{2} \cosh ^{2} x+\lambda^{2}}{2} v\right) \\
& \times \mathbb{E}\left[f(\beta(v)) e^{\mu \sinh x \cdot \beta(v)}\right] \\
& =\int_{\mathbb{R}} d y e^{-\left(\sqrt{\mu^{2} \cosh ^{2} x+\lambda^{2}} \cosh y-\mu \sinh x \sinh y\right)} f(\sinh y),
\end{aligned}
$$

where we used the change of the variables with $e^{y} / \mu=v$ as well as the Cameron-Martin formula applied to $\mu \sinh x \cdot v+\beta(v)$ for the second equality and Proposition 1.1 for the third. Thanks to (1.16), we may rewrite

$$
\begin{aligned}
& e^{-\left(\sqrt{\mu^{2} \cosh ^{2} x+\lambda^{2}} \cosh y-\mu \sinh x \sinh y\right)} \\
& =e^{-\mu \cosh (y-x)} e^{-\left(\sqrt{\mu^{2} \cosh ^{2} x+\lambda^{2}}-\mu \cosh x\right) \cosh y} \\
& =e^{-\mu \cosh (y-x)} \mathbb{E}\left[\exp \left\{-\frac{\lambda^{2}}{2} \tau_{\cosh y}\left(B^{(\mu \cosh x)}\right)\right\}\right] .
\end{aligned}
$$

We plug the last expression into the rightmost side of (2.32). After translating the variable $y$ by $x$, we use Fubini's theorem to arrive at the identity

$$
\begin{aligned}
& \mathbb{E}\left[f\left(e^{z_{\mu}} \sinh x+\beta\left(e^{z_{\mu}} / \mu\right)\right) \exp \left(-\frac{\lambda^{2}}{2} \frac{e^{z_{\mu}}}{\mu}\right)\right] \\
& =\mathbb{E}\left[f\left(\sinh \left(x+z_{\mu}\right)\right) \exp \left\{-\frac{\lambda^{2}}{2} \tau_{\cosh \left(x+z_{\mu}\right)}\left(\hat{B}^{(\mu \cosh x)}\right)\right\}\right] .
\end{aligned}
$$

Here on the right-hand side, $\hat{B}$ denotes a Brownian motion independent of $z_{\mu}$. As $f$ and $\lambda$ are arbitrary, we obtain the identity (2.15) as claimed. Proof of Proposition 2.3 is complete.

We close this subsection by listing some facts deduced from Proposition 2.3. Remark 2.4. (1) If we let $\mu \rightarrow \infty$, the identity (2.13) remains valid in the sense that as two-dimensional random variables, both sides of (2.13) converge in law to ( $\sinh x, C \cosh x)$ by Proposition 2.1.

(2) It follows readily from Proposition 2.1 that $\sinh z_{\mu}$ converges in law to 0 as $\mu \rightarrow \infty$, which may be strengthened as convergence in law of $\sqrt{\mu} \sinh z_{\mu}$ to the standard normal distribution. Indeed, by the scaling property of Brownian motion and by (2.13) (or 
$(2.5))$,

$$
\begin{aligned}
\beta\left(e^{z_{\mu}}\right) & \stackrel{(d)}{=} \sqrt{\mu} \beta\left(e^{z_{\mu}} / \mu\right) \\
& \stackrel{(d)}{=} \sqrt{\mu} \sinh z_{\mu}
\end{aligned}
$$

the leftmost side of which converges in law to $\beta(1)$ as $\mu \rightarrow \infty$ thanks to Proposition 2.1. We may rephrase this convergence in law as that of $(\sqrt{\mu} / 2)\left(I_{\sqrt{\mu}, \sqrt{\mu}}^{(0)}-1 / I_{\sqrt{\mu}, \sqrt{\mu}}^{(0)}\right)$ in view of (2.9). The same convergence also holds true for any $\operatorname{GIG}(\nu ; \sqrt{\mu}, \sqrt{\mu})$ laws; in fact, a simple change of the variables shows that for every $\nu \in \mathbb{R}$ and for any bounded measurable function $f$ on $\mathbb{R}$,

$$
\begin{aligned}
& \mathbb{E}\left[f\left((\sqrt{\mu} / 2)\left(I_{\sqrt{\mu}, \sqrt{\mu}}^{(\nu)}-1 / I_{\sqrt{\mu}, \sqrt{\mu}}^{(\nu)}\right)\right)\right] \\
& =\frac{1}{2 \sqrt{\mu} e^{\mu} K_{\nu}(\mu)} \int_{\mathbb{R}} \frac{d x}{\sqrt{1+x^{2} / \mu}}\left(\frac{x}{\sqrt{\mu}}+\sqrt{1+\frac{x^{2}}{\mu}}\right)^{\nu} \exp \left(-\frac{x^{2}}{1+\sqrt{1+x^{2} / \mu}}\right) f(x),
\end{aligned}
$$

which converges to $\mathbb{E}[f(\beta(1))]$ as $\mu \rightarrow \infty$ due to (2.11). In [14], a similar computation reveals the convergence in law of $\sqrt{\mu}\left(I_{\sqrt{\mu}, \sqrt{\mu}}^{(\nu)}-1\right)$ to the standard normal distribution, from which the same convergence of $\sqrt{\mu} \log \left(I_{\sqrt{\mu}, \sqrt{\mu}}^{(\nu)}\right)$ is also deduced; see Equations (3.10) and (3.11) of the above-cited reference.

(3) As for each side of the identity (2.13), if we divide the second coordinate by the square root of the sum of 1 and square of the first coordinate, then we obtain the identity in law:

$$
\frac{C e^{z_{\mu}} \cosh x+\hat{\beta}\left(e^{z_{\mu}} / \mu\right)}{\sqrt{1+\left(e^{z_{\mu}} \sinh x+\beta\left(e^{z_{\mu}} / \mu\right)\right)^{2}}} \stackrel{(d)}{=} C,
$$

which holds for every fixed $\mu>0$ and $x \in \mathbb{R}$. The above identity may also be deduced from (1.18) by taking $\tau=\tau_{1 / \mu}(Z)$, which is finite a.s. because of the transience of $Z$ noted in Remark 2.3(1).

(4) For any $x, y \in \mathbb{R}$, there holds the identity in law

$$
\sinh \left(y+z_{\mu}\right) \stackrel{(d)}{=} \sinh \left(x+z_{\mu}\right)+\mu(\sinh y-\sinh x) \tau_{\cosh \left(x+z_{\mu}\right)}\left(\hat{B}^{(\mu \cosh x)}\right)
$$

with $\hat{B}$ a Brownian motion independent of $z_{\mu}$ on the right-hand side; indeed,

$$
\begin{aligned}
\sinh \left(y+z_{\mu}\right) & \stackrel{(d)}{=} e^{z_{\mu}} \sinh y+\beta\left(e^{z_{\mu}} / \mu\right) \\
& =e^{z_{\mu}}(\sinh y-\sinh x)+e^{z_{\mu}} \sinh x+\beta\left(e^{z_{\mu}} / \mu\right) \\
& \stackrel{(d)}{=} \mu \tau_{\cosh \left(x+z_{\mu}\right)}\left(\hat{B}^{(\mu \cosh x)}\right) \cdot(\sinh y-\sinh x)+\sinh \left(x+z_{\mu}\right)
\end{aligned}
$$

where the third line is due to (2.15). 


\subsection{Proof of Theorem 1.3}

In this subsection we prove Theorem 1.3, whose assertion is nothing but a rewriting of the following one: as in the statement of the theorem, $\varepsilon$ denotes a Rademacher variable.

Proposition 2.4. Suppose that $C$ and $\varepsilon$ are independent. Then it holds that:

(i) for every $x, y \in \mathbb{R}$,

$$
\sinh (\operatorname{Argsh}(C \cosh x)+y \varepsilon) \stackrel{(d)}{=} C \cosh (x+y \varepsilon)
$$

(ii) for every $a \in[-1,1]$ and $y \in \mathbb{R}$,

$$
\sinh (\operatorname{Argsh}(a C)+y \varepsilon) \stackrel{(d)}{=} a C \cosh y+\sqrt{1-a^{2}} \sinh (y \varepsilon) .
$$

Since we will use Lemma 2.1 in the case $\lambda=0$ below, we repeat it here for the reader's convenience, putting $\lambda=0$ : for every $\mu>0$ and $\xi \in \mathbb{R}$, it holds that

$$
\int_{\mathbb{R}} d y e^{-\mu \cosh y} e^{i \xi \sinh (x+y)}=\int_{0}^{\infty} \frac{d v}{v} \exp \left(-\frac{1}{2 v}\right) \exp \left(-\frac{\mu^{2}+\xi^{2}}{2} v\right) e^{i \mu \xi v \sinh x}
$$

for all $x \in \mathbb{R}$.

Proof of Proposition 2.4. We start with the proof of (i). We replace $x$ in (2.33) by $\operatorname{Argsh}(C \cosh x)$ and take the expectation on both sides with respect to $C$. Then by Fubini's theorem, we have

$$
\begin{aligned}
& \int_{\mathbb{R}} d y e^{-\mu \cosh y} \mathbb{E}[\exp \{i \xi \sinh (\operatorname{Argsh}(C \cosh x)+y)\}] \\
& =\int_{0}^{\infty} \frac{d v}{v} \exp \left(-\frac{1}{2 v}\right) \exp \left(-\frac{\mu^{2}+\xi^{2}+2 \mu|\xi| \cosh x}{2} v\right)
\end{aligned}
$$

thanks to the identity $\mathbb{E}\left[e^{i \mu \xi v C \cosh x}\right]=\exp (-\mu|\xi| v \cosh x)$ for $v>0$. Rewriting

$$
\begin{aligned}
\exp \left(-\frac{\mu^{2}+\xi^{2}+2 \mu|\xi| \cosh x}{2} v\right) & =\exp \left\{-\frac{(\mu+|\xi| \cosh x)^{2}}{2} v+\frac{\xi^{2}}{2} v \sinh ^{2} x\right\} \\
& =\exp \left\{-\frac{(\mu+|\xi| \cosh x)^{2}}{2} v\right\} \mathbb{E}\left[e^{-|\xi| \beta(v) \sinh x}\right]
\end{aligned}
$$

with $\beta$ a Brownian motion, we see from Proposition 1.1 that the right-hand side of (2.34) is equal to

$$
\int_{\mathbb{R}} d y e^{-(\mu+|\xi| \cosh x) \cosh y} e^{-|\xi| \sinh x \sinh y}=\int_{\mathbb{R}} d y e^{-\mu \cosh y} e^{-|\xi| \cosh (x+y)} .
$$


Replacing $e^{-|\xi| \cosh (x+y)}$ in the last integral by $\mathbb{E}[\exp \{i \xi C \cosh (x+y)\}]$, we obtain from (2.34) the identity

$$
\begin{aligned}
& \int_{\mathbb{R}} d y e^{-\mu \cosh y} \mathbb{E}[\exp \{i \xi \sinh (\operatorname{Argsh}(C \cosh x)+y)\}] \\
& =\int_{\mathbb{R}} d y e^{-\mu \cosh y} \mathbb{E}[\exp \{i \xi C \cosh (x+y)\}]
\end{aligned}
$$

which holds for every $\mu>0, \xi \in \mathbb{R}$ and $x \in \mathbb{R}$. Now we observe that for any bounded measurable function $f: \mathbb{R} \rightarrow \mathbb{R}$,

$$
\begin{aligned}
\int_{\mathbb{R}} d y e^{-\mu \cosh y} f(y) & =2 \int_{0}^{\infty} d y e^{-\mu \cosh y} \mathbb{E}[f(\varepsilon y)] \\
& =2 \int_{1}^{\infty} \frac{d u}{\sqrt{u^{2}-1}} e^{-\mu u} \mathbb{E}[f(\varepsilon \operatorname{Argch} u)],
\end{aligned}
$$

where the second line is due to change of the variables with $y=\operatorname{Argch} u, u>1$. Here $\operatorname{Argch} u=\log \left(u+\sqrt{u^{2}-1}\right)$. Thanks to the above observation as well as to the injectivity of Laplace transform, we conclude from (2.35) that

$$
\begin{aligned}
& \mathbb{E}[\exp \{i \xi \sinh (\operatorname{Argsh}(C \cosh x)+\varepsilon \operatorname{Argch} u)\}] \\
& =\mathbb{E}[\exp \{i \xi C \cosh (x+\varepsilon \operatorname{Argch} u)\}]
\end{aligned}
$$

for any $u \geq 1$, where $\varepsilon$ is assumed to be independent of $C$ on both sides. The assertion (i) of the proposition then follows readily since $\xi$ is arbitrary and $\varepsilon$ is symmetric.

Proof of the assertion (ii) is done similarly to the above. For each fixed $a \in[-1,1]$, we replace $x$ in (2.33) by $\operatorname{Argsh}(a C)$ and take the expectation on both sides to get the equality

$$
\begin{aligned}
& \int_{\mathbb{R}} d y e^{-\mu \cosh y} \mathbb{E}[\exp \{i \xi \sinh (\operatorname{Argsh}(a C)+y)\}] \\
& =\int_{0}^{\infty} \frac{d v}{v} \exp \left(-\frac{1}{2 v}\right) \exp \left\{-\frac{(\mu+|a \xi|)^{2}}{2} v\right\} \exp \left\{-\frac{\xi^{2}}{2}\left(1-a^{2}\right) v\right\} .
\end{aligned}
$$

By noting that

$$
\exp \left\{-\frac{\xi^{2}}{2}\left(1-a^{2}\right) v\right\}=\mathbb{E}\left[\exp \left\{i \xi \sqrt{1-a^{2}} \beta(v)\right\}\right]
$$

with $\beta$ a Brownian motion, and by using Proposition 1.1, the right-hand side of (2.36) is rewritten as

$$
\begin{aligned}
& \int_{\mathbb{R}} d y e^{-(\mu+|a \xi|) \cosh y} e^{i \xi \sqrt{1-a^{2}} \sinh y} \\
& =\int_{\mathbb{R}} d y e^{-\mu \cosh y} \mathbb{E}\left[\exp \left\{i \xi\left(a C \cosh y+\sqrt{1-a^{2}} \sinh y\right)\right\}\right] .
\end{aligned}
$$

Rest of the proof proceeds in the same way as in the latter half of the proof of (i) by noting the symmetry of the function $\cosh y, y \in \mathbb{R}$. 
We are ready to prove Theorem 1.3 ,

Proof of Theorem 1.3. The assertion (i) of Proposition 2.4 entails that by symmetry of $\varepsilon$

$$
a C \cosh y+\sqrt{1+a^{2} C^{2}} \varepsilon \sinh y \stackrel{(d)}{=} C\left(a \cosh y+\sqrt{a^{2}-1} \varepsilon \sinh y\right)
$$

for any $y \in \mathbb{R}$. Here we write $a=\cosh x \geq 1$. Dividing both sides by $\cosh y$, we see that the assertion (i) of the theorem holds true for any $\theta \in(-1,1)$, and hence for any $\theta \in[-1,1]$ since it is clear that both sides of the claimed identity are continuous in $\theta$ with respect to the topology of weak convergence. Extension to the case $a \leq-1$ is done by noting that $(C,-\varepsilon)$ and $(-C, \varepsilon)$, as well as $(-C,-\varepsilon)$, are identical in law with $(C, \varepsilon)$ due to independence of $C$ and $\varepsilon$. This completes the proof of the assertion (i). We omit the proof of (ii) because it proceeds quite similarly.

We close this section with a remark on Theorem 1.3 as well as on the integral identity (2.33) used in the proof of Proposition 2.4.

Remark 2.5. (1) For every fixed $\theta \in[-1,1]$, Theorem 1.3 entails that the characteristic function of $a C+\theta \sqrt{1+a^{2} C^{2}} \varepsilon$ is given by

$$
\exp (-|a \xi|) \cosh \left(\theta \xi \sqrt{a^{2}-1}\right), \quad \xi \in \mathbb{R}
$$

in the case $|a| \geq 1$ while it is given by

$$
\exp (-|a \xi|) \cos \left(\theta \xi \sqrt{1-a^{2}}\right), \quad \xi \in \mathbb{R}
$$

in the case $|a| \leq 1$. To verify the expression in the former case, it suffices to deduce from Theorem $1.3(\mathrm{i})$ that for any $\xi \in \mathbb{R}$,

$$
\mathbb{E}\left[\exp \left\{i \xi\left(a C+\theta \sqrt{1+a^{2} C^{2}} \varepsilon\right)\right\}\right]=\mathbb{E}\left[\exp \left\{-|\xi|\left|a+\theta \sqrt{a^{2}-1} \varepsilon\right|\right\}\right]
$$

and that since $|\theta| \leq 1$ and $|\varepsilon|=1$ a.s.,

$$
\left|a+\theta \sqrt{a^{2}-1} \varepsilon\right|=|a|+\theta \sqrt{a^{2}-1} \varepsilon \operatorname{sgn} a \quad \text { a.s. }
$$

where $\operatorname{sgn} a$ is the signature of $a$. The above expressions of characteristic functions in two cases suggest that by means of analytic continuation, the assertion (ii) of the theorem may be seen as a consequence of the assertion (i) or vice versa.

(2) It is of interest to observe from (2.33) that if we take $\xi$ positive, then the multivariate function

$$
\int_{\mathbb{R}} d y e^{-\mu \cosh y} e^{i \xi \sinh (x+y)}, \quad \mu, \xi>0, x \in \mathbb{R}
$$


is symmetric with respect to $\mu$ and $\xi$. As will be seen in Subsection 3.2 below, this fact yields the following symmetry concerning the laws of functionals of the form $e^{2 B_{t}} v+$ $A_{t}, v>0$, for every $t>0$ :

$$
\begin{aligned}
& \frac{1}{v} \exp \left(-\frac{1}{2 v}\right) \mathbb{P}\left(e^{2 B_{t}} v+A_{t} \in d u\right) d v \\
& =\frac{1}{u} \exp \left(-\frac{1}{2 u}\right) \mathbb{P}\left(e^{2 B_{t}} u+A_{t} \in d v\right) d u, \quad u, v>0 .
\end{aligned}
$$

We may replace $t$ by any positive and finite stopping time $\tau$ of the process $Z$.

\section{Some related results}

In this section, we present several results relevant to our discussions developed in the previous section.

\subsection{Derivations of (1.9) in part and Dufresne's identity}

This subsection is concerned with partial derivations of the identity (1.9) in Theorem 1.1, one of which is to be applied to a proof of an identity in law due to Dufresne [9].

For each $\nu \in \mathbb{R}$, we set

$$
A_{t}^{(\nu)}:=\int_{0}^{t} e^{2 B_{s}^{(\nu)}} d s, \quad t \geq 0
$$

where $B^{(\nu)}$ is the Brownian motion with drift $\nu$. Dufresne's identity ([9, Proposition 4.4.4(b)]) asserts that when $\nu>0$, the perpetual integral $A_{\infty}^{(-\nu)}=\int_{0}^{\infty} e^{2 B_{s}^{(-\nu)}} d s$ is identical in law with the reciprocal of twice of a gamma variable with parameter $\nu$ :

$$
\begin{gathered}
A_{\infty}^{(-\nu)} \stackrel{(d)}{=} \frac{1}{2 \gamma_{\nu}}, \\
\mathbb{P}\left(\gamma_{\nu} \in d v\right)=\frac{1}{\Gamma(\nu)} v^{\nu-1} e^{-v} d v, \quad v>0,
\end{gathered}
$$

where $\Gamma$ is the gamma function; we refer to Theorem 6.2 and Proposition 6.3 in 23 . for different proofs of (3.1) than the original one.

In this subsection, we use the invariance formulae for Cauchy variable in the form Proposition 2.4 to see that the identity (1.9) is recovered partly in two ways; we then apply one of those partial identities to give another proof of Dufresne's identity (3.1). Our argument to be developed here does not use Proposition 2.2 and relies only on Proposition 2.4 and the identity (1.2), which makes the contents of this subsection completely self-contained.

In the proposition exhibited below, the first identity (3.3) treats the one between second coordinates in (1.9) when $\tau=t$, and the second identity (3.4) treats the case $x=0$ and $\tau=t$ in (1.9) with third coordinates dropped. 
Proposition 3.1. Under the same setting as in Theorem 1.1, it holds that for every fixed $t>0$ and $x \in \mathbb{R}$,

$$
C e^{B_{t}} \cosh x+\hat{\beta}\left(A_{t}\right) \stackrel{(d)}{=} C \cosh \left(x+B_{t}\right),
$$

and that for every fixed $t>0$,

$$
\left(\beta\left(A_{t}\right), C e^{B_{t}}+\hat{\beta}\left(A_{t}\right)\right) \stackrel{(d)}{=}\left(\sinh B_{t}, C \cosh B_{t}\right) .
$$

Proof. For three independent elements $B, C$ and a Rademacher variable $\varepsilon$, we substitute $y$ in Proposition 2.4(i) by $B_{t}$ to obtain

$$
\sinh \left(\operatorname{Argsh}(C \cosh x)+B_{t}\right) \stackrel{(d)}{=} C \cosh \left(x+B_{t}\right)
$$

where we used the fact that $\varepsilon B_{t} \stackrel{(d)}{=} B_{t}$ by independence of $B$ and $\varepsilon$ and by symmetry of $B$. In virtue of the identity (1.2), the left-hand side of the above identity is identical in law with

$$
C e^{B_{t}} \cosh x+\hat{\beta}\left(A_{t}\right),
$$

which shows (3.3).

To prove (3.4), we repeat the same argument as above to obtain from Proposition 2.4(ii),

$$
a C e^{B_{t}}+\beta\left(A_{t}\right) \stackrel{(d)}{=} a C \cosh B_{t}+\sqrt{1-a^{2}} \sinh B_{t}
$$

for every $a \in[-1,1]$, where on the left-hand side, $\beta$ is a Brownian motion independent of $B$ and $C$. Taking the Fourier transform on both sides, we have for any $\xi \in \mathbb{R}$,

$$
\mathbb{E}\left[\exp \left(-|a \xi| e^{B_{t}}-\frac{\xi^{2}}{2} A_{t}\right)\right]=\mathbb{E}\left[\exp \left(i a \xi C \cosh B_{t}\right) \exp \left(i \xi \sqrt{1-a^{2}} \sinh B_{t}\right)\right] .
$$

We may express the left-hand side of (3.5) as

$$
\mathbb{E}\left[\exp \left\{i a \xi\left(C e^{B_{t}}+\hat{\beta}\left(A_{t}\right)\right)\right\} \exp \left\{i \xi \sqrt{1-a^{2}} \beta\left(A_{t}\right)\right\}\right]
$$

in terms of the pair of random variables on the left-hand side of (3.4). Since the last two expressions agree for any $a$ and $\xi$, the second identity (3.4) also follows.

Using the former identity (3.3) in Proposition 3.1, we are going to prove

Proposition 3.2. Let $\nu>0$ and fix $t>0$. Then it holds that

$$
\frac{e^{2 B_{t}^{(-\nu)}}}{2 \gamma_{\nu}}+A_{t}^{(-\nu)} \stackrel{(d)}{=} \frac{1}{2 \gamma_{\nu}},
$$

where on the left-hand side, the gamma variable $\gamma_{\nu}$ with parameter $\nu$ is independent of B. In particular, letting $t \rightarrow \infty$ on the left-hand side leads to (3.1). 
Remark 3.1. It should be noted that Dufresne's identity (3.1) also implies (3.6); indeed, for every $t>0$,

$$
\begin{aligned}
A_{\infty}^{(-\nu)} & =A_{t}^{(-\nu)}+\int_{t}^{\infty} e^{2 B_{s}^{(-\nu)}} d s \\
& =A_{t}^{(-\nu)}+e^{2 B_{t}^{(-\nu)}} \int_{0}^{\infty} e^{2\left(B_{s+t}^{(-\nu)}-B_{t}^{(-\nu)}\right)} d s
\end{aligned}
$$

the last integral being independent of $B_{s}^{(-\nu)}, 0 \leq s \leq t$, and by (3.1), distributed as $1 /\left(2 \gamma_{\nu}\right)$.

Notice that by the same reasoning as used in the proof of the identity (2.14) in Proposition 2.3, the identity (3.3) admits the equivalent expression

$$
\tau_{e^{B_{t}} \cosh x}(\hat{B})+A_{t} \stackrel{(d)}{=} \tau_{\cosh \left(x+B_{t}\right)}(\hat{B})
$$

as in (1.10). Here $\hat{B}$ is a Brownian motion independent of $B$ on both sides. We start the proof of Proposition 3.2 from (3.7). We also observe from (3.2) and another integral representation (2.8) of the Macdonald function that when $\nu>0$, we may put that representation into the form

$$
K_{\nu}(z)=2^{\nu-1} \Gamma(\nu) z^{-\nu} \mathbb{E}\left[\exp \left(-\frac{z^{2}}{4 \gamma_{\nu}}\right)\right], \quad z>0
$$

Proof of Proposition 3.2. Recalling (1.16) and replacing $\lambda$ therein by every $z>0$, we take the Laplace transform on both sides of (3.7) to get

$$
\mathbb{E}\left[\exp \left(-z e^{B_{t}} \cosh x-\frac{z^{2}}{2} A_{t}\right)\right]=\mathbb{E}\left[\exp \left\{-z \cosh \left(x+B_{t}\right)\right\}\right]
$$

For each fixed $\nu \in \mathbb{R}$, we integrate both sides over $\mathbb{R}$ with respect to $(1 / 2) e^{-\nu x} d x$. Then by Fubini's theorem and by the integral representation (2.4) of $K_{\nu}$, the left-hand side becomes

$$
\mathbb{E}\left[K_{\nu}\left(z e^{B_{t}}\right) \exp \left(-\frac{z^{2}}{2} A_{t}\right)\right]
$$

As for the right-hand side, by Fubini's theorem, we compute

$$
\begin{aligned}
\frac{1}{2} \mathbb{E}\left[\int_{\mathbb{R}} d x e^{-\nu x} \exp \left\{-z \cosh \left(x+B_{t}\right)\right\}\right] & =\frac{1}{2} \mathbb{E}\left[\int_{\mathbb{R}} d x e^{-\nu\left(x-B_{t}\right)} \exp (-z \cosh x)\right] \\
& =\frac{1}{2} \int_{\mathbb{R}} d x e^{-z \cosh x} e^{-\nu x} \times \mathbb{E}\left[e^{\nu B_{t}}\right] \\
& =K_{\nu}(z) e^{\nu^{2} t / 2} .
\end{aligned}
$$


We let $\nu>0$ hereafter. Assuming that $\gamma_{\nu}$ is independent of $B$, we now use (3.8) to rewrite $(3.10)$ as

$$
\begin{aligned}
& 2^{\nu-1} \Gamma(\nu) z^{-\nu} \mathbb{E}\left[e^{-\nu B_{t}} \exp \left\{-\frac{z^{2}}{2}\left(\frac{e^{2 B_{t}}}{2 \gamma_{\nu}}+A_{t}\right)\right\}\right] \\
& =2^{\nu-1} \Gamma(\nu) z^{-\nu} \mathbb{E}\left[\exp \left\{-\frac{z^{2}}{2}\left(\frac{e^{2 B_{t}^{(-\nu)}}}{2 \gamma_{\nu}}+A_{t}^{(-\nu)}\right)\right\}\right] e^{\nu^{2} t / 2} .
\end{aligned}
$$

Here we used the Cameron-Martin formula for the equality. Since the last expression agrees with (3.11), we have for any $z>0$,

$$
\mathbb{E}\left[\exp \left\{-\frac{z^{2}}{2}\left(\frac{e^{2 B_{t}^{(-\nu)}}}{2 \gamma_{\nu}}+A_{t}^{(-\nu)}\right)\right\}\right]=\mathbb{E}\left[\exp \left(-\frac{z^{2}}{2} \cdot \frac{1}{2 \gamma_{\nu}}\right)\right]
$$

thanks to (3.8). Therefore the identity (3.6) is proven by the injectivity of Laplace transform. Letting $t \rightarrow \infty$ on the left-hand side of (3.12) also proves (3.1) by the bounded convergence theorem.

Remark 3.2. (1) Recalling the well-known fact, as readily seen from (2.2) and the identity $B_{1}^{2} \stackrel{(d)}{=} 2 \gamma_{1 / 2}$, that

$$
\tau_{a}(B) \stackrel{(d)}{=} \frac{a^{2}}{2 \gamma_{1 / 2}}
$$

for every $a \in \mathbb{R}$, we may rephrase (3.7) as

$$
\frac{e^{2 B_{t}} \cosh ^{2} x}{2 \gamma_{1 / 2}}+A_{t} \stackrel{(d)}{=} \frac{\cosh ^{2}\left(x+B_{t}\right)}{2 \gamma_{1 / 2}},
$$

which holds for every fixed $t>0$ and $x \in \mathbb{R}$. Here $B$ and $\gamma_{1 / 2}$ are independent on both sides. For each fixed $t$, the family of identities (3.13) indexed by $x \in \mathbb{R}$, may be regarded as an equivalent expression for that of identities (3.6) indexed by $\nu>0$ because reasoning used in the above proof of Proposition 3.2 is invertible.

(2) For every $t>0$, by adopting the identity

$$
\left(C e^{B_{t}} \cosh x+\hat{\beta}\left(A_{t}\right), Z_{t}\right) \stackrel{(d)}{=}\left(C \cosh \left(x+B_{t}\right), Z_{t}\right)
$$

from (1.9), the identity (3.6) extends to

$$
\left(\frac{e^{2 B_{t}^{(-\nu)}}}{2 \gamma_{\nu}}+A_{t}^{(-\nu)}, e^{-B_{t}^{(-\nu)}} A_{t}^{(-\nu)}\right) \stackrel{(d)}{=}\left(\frac{1}{2 \gamma_{\nu}}, e^{-B_{t}^{(-\nu)} A_{t}^{(-\nu)}}\right),
$$

where $B$ and $\gamma_{\nu}$ are independent on both sides. In fact, by (3.14), it holds that for any bounded measurable function $f: \mathbb{R} \rightarrow \mathbb{R}$,

$$
\mathbb{E}\left[f\left(Z_{t}\right) \exp \left(-z e^{B_{t}} \cosh x-\frac{z^{2}}{2} A_{t}\right)\right]=\mathbb{E}\left[f\left(Z_{t}\right) \exp \left\{-z \cosh \left(x+B_{t}\right)\right\}\right],
$$


to which the same reasoning as in the proof of Proposition 3.2 applies. The extension (3.15) may also be seen as an immediate consequence of [21, Proposition 13.1] together with [20, Proposition 1.7]. In view of those two propositions, the above identity (3.15) remains true when we replace $t$ by any positive and finite stopping time of the process $\left\{e^{-B_{t}^{(-\nu)} A_{t}^{(-\nu)}}\right\}_{t>0}$.

(3) As a consequence of one of their results relevant to hyperbolic Bessel processes, the equality (3.9) is obtained in Proposition 2.4 of [13] by Jakubowski and Wiśniewolski, who also give an alternative proof of Bougerol's identity (1.1) based on their study.

We close this subsection with a representation for the joint Laplace transform of the law of $\left(e^{B_{t}}, A_{t}\right)$ in terms of $B_{t}$, which is easily deduced from proofs of Propositions 3.1 and 3.2, and which may be compared with Remark 2.5(1).

Proposition 3.3. Let $\lambda \geq 0$ and $\xi \in \mathbb{R}$. Then for every $t>0$, the expectation

$$
\mathbb{E}\left[\exp \left(-\lambda e^{B_{t}}-\frac{\xi^{2}}{2} A_{t}\right)\right]
$$

admits the following representation:

$$
\mathbb{E}\left[\exp \left(-\lambda \cosh B_{t}\right) \cos \left(\sqrt{\xi^{2}-\lambda^{2}} \sinh B_{t}\right)\right] \quad \text { if } \lambda \leq|\xi|,
$$

and

$$
\mathbb{E}\left[\exp \left(-\lambda \cosh B_{t}\right) \cosh \left(\sqrt{\lambda^{2}-\xi^{2}} \sinh B_{t}\right)\right] \quad \text { if } \lambda \geq|\xi|
$$

Proof. We recall from the proof of Proposition 3.1 the equality (3.5), which may be restated, by symmetry of $\sinh B_{t}$, as

$$
\mathbb{E}\left[\exp \left(-|a \xi| e^{B_{t}}-\frac{\xi^{2}}{2} A_{t}\right)\right]=\mathbb{E}\left[\exp \left(-|a \xi| \cosh B_{t}\right) \cos \left(\xi \sqrt{1-a^{2}} \sinh B_{t}\right)\right] .
$$

Here $a \in[-1,1]$ and $\xi \in \mathbb{R}$ are arbitrary. Putting $|a \xi|=\lambda \leq|\xi|$ shows (3.16). To see (3.17), we recall from the proof of Proposition 3.2 the equality (3.9), which asserts that for every $z \geq 0$,

$$
\begin{aligned}
& \mathbb{E}\left[\exp \left(-z e^{B_{t}} \cosh x-\frac{z^{2}}{2} A_{t}\right)\right] \\
& =\mathbb{E}\left[\exp \left(-z \cosh x \cosh B_{t}-z \sinh x \sinh B_{t}\right)\right] \\
& =\mathbb{E}\left[\exp \left(-z \cosh x \cosh B_{t}\right) \cosh \left(z \sinh x \sinh B_{t}\right)\right],
\end{aligned}
$$

where the second equality is due to symmetry of $\sinh B_{t}$. Writing $z \cosh x=\lambda$ and $z=|\xi|$ leads to (3.17) and finishes the proof. 
With the help of Theorem 1.1, we may replace $t$ in the statement of Proposition 3.3 by any positive and finite stopping time $\tau$ of $Z$; in fact, similarly to Proposition 3.3, we see from Proposition 2.3 (i) that for every $\mu>0$,

$$
\begin{aligned}
& \mathbb{E}\left[\exp \left(-\lambda e^{z_{\mu}}-\frac{\xi^{2}}{2} \frac{e^{z_{\mu}}}{\mu}\right)\right] \\
& = \begin{cases}\mathbb{E}\left[\exp \left(-\lambda \cosh z_{\mu}\right) \cos \left(\sqrt{\xi^{2}-\lambda^{2}} \sinh z_{\mu}\right)\right] & \text { if } \lambda \leq|\xi|, \\
\mathbb{E}\left[\exp \left(-\lambda \cosh z_{\mu}\right) \cosh \left(\sqrt{\lambda^{2}-\xi^{2}} \sinh z_{\mu}\right)\right] & \text { if } \lambda \geq|\xi|,\end{cases}
\end{aligned}
$$

and if we integrate both sides with respect to $\mathbb{P}\left(1 / Z_{\tau} \in d \mu\right)$, then the above-mentioned replacement is verified thanks to Proposition 2.2. Note that by using (2.9) and (2.10), the left-hand side of (3.18) is computed, regardless of whether $\lambda \leq|\xi|$ or not, as

$$
\frac{K_{0}\left(\sqrt{(\mu+\lambda)^{2}+\xi^{2}-\lambda^{2}}\right)}{K_{0}(\mu)}
$$

which also reveals the equality

$$
\mathbb{E}\left[\exp \left(-\lambda e^{B_{\tau}}-\frac{\xi^{2}}{2} A_{\tau}\right)\right]=\mathbb{E}\left[\frac{K_{0}\left(\sqrt{\left(1 / Z_{\tau}+\lambda\right)^{2}+\xi^{2}-\lambda^{2}}\right)}{K_{0}\left(1 / Z_{\tau}\right)}\right] .
$$

When $\tau=t>0$, these expectations are expressed as

$$
2 \int_{0}^{\infty} \frac{d \mu}{\mu} K_{0}\left(\sqrt{(\mu+\lambda)^{2}+\xi^{2}-\lambda^{2}}\right) \Theta_{\mu}(t)
$$

owing to (2.26). We will return to the last expression in Subsection A.2 of the appendix.

\subsection{A symmetry for laws of $e^{2 B_{\tau}} v+A_{\tau}, v>0$}

In this subsection, we prove

Proposition 3.4. Let $\tau$ be a stopping time of the process $Z$ such that $0<\tau<\infty$ a.s. Then there takes place the coincidence

$$
\begin{aligned}
& \frac{1}{v} \exp \left(-\frac{1}{2 v}\right) \mathbb{P}\left(e^{2 B_{\tau}} v+A_{\tau} \in d u, Z_{\tau} \in d w\right) d v \\
& =\frac{1}{u} \exp \left(-\frac{1}{2 u}\right) \mathbb{P}\left(e^{2 B_{\tau}} u+A_{\tau} \in d v, Z_{\tau} \in d w\right) d u
\end{aligned}
$$

as measures on $(0, \infty)^{3}$.

Proof. Observe from (2.33) that for any $\mu, \xi>0$, we have

$$
\int_{\mathbb{R}} d y e^{-\mu \cosh y} e^{i \xi \sinh (x+y)}=\int_{\mathbb{R}} d y e^{-\xi \cosh y} e^{i \mu \sinh (x+y)}
$$


for all $x \in \mathbb{R}$. Let $f:(0, \infty) \rightarrow \mathbb{R}$ be bounded and measurable. Upon substituting $x$ by $B_{\tau}$ in (3.21), we multiply both sides by $f\left(Z_{\tau}\right)$ and take the expectation to obtain

$$
\begin{aligned}
& \int_{\mathbb{R}} d y e^{-\mu \cosh y} \mathbb{E}\left[f\left(Z_{\tau}\right) \exp \left\{i \xi \sinh \left(y+B_{\tau}\right)\right\}\right] \\
& =\int_{\mathbb{R}} d y e^{-\xi \cosh y} \mathbb{E}\left[f\left(Z_{\tau}\right) \exp \left\{i \mu \sinh \left(y+B_{\tau}\right)\right\}\right]
\end{aligned}
$$

by Fubini's theorem. Recalling from Theorem 1.1, as well as from Theorem 1.2, the identity

$$
\left(\sinh \left(y+B_{\tau}\right), Z_{\tau}\right) \stackrel{(d)}{=}\left(e^{B_{\tau}} \sinh y+\beta\left(A_{\tau}\right), Z_{\tau}\right)
$$

for every $y \in \mathbb{R}$, we rewrite the left-hand side of (3.22) as

$$
\begin{aligned}
& \int_{\mathbb{R}} d y e^{-\mu \cosh y} \mathbb{E}\left[f\left(Z_{\tau}\right) e^{i \xi e^{B} \tau \sinh y} \exp \left(-\frac{\xi^{2}}{2} A_{\tau}\right)\right] \\
& =\int_{0}^{\infty} \frac{d v}{v} \exp \left(-\frac{1}{2 v}\right) \exp \left(-\frac{\mu^{2}}{2} v\right) \mathbb{E}\left[f\left(Z_{\tau}\right) e^{i \xi e^{B \tau} \beta(v)} \exp \left(-\frac{\xi^{2}}{2} A_{\tau}\right)\right] \\
& =\int_{0}^{\infty} \frac{d v}{v} \exp \left(-\frac{1}{2 v}\right) \exp \left(-\frac{\mu^{2}}{2} v\right) \mathbb{E}\left[f\left(Z_{\tau}\right) \exp \left\{-\frac{\xi^{2}}{2}\left(e^{2 B_{\tau}} v+A_{\tau}\right)\right\}\right],
\end{aligned}
$$

where, $\beta$ being a Brownian motion independent of $B$, we used Proposition 1.1 for the second line. Since the last integral agrees, by (3.22), with the one in which $\mu$ and $\xi$ are exchanged, we obtain the statement of the proposition thanks to arbitrariness of $f, \mu$ and $\xi$.

Remark 3.3. When $\tau$ is only assumed to be finite a.s., the relation (3.20) is valid by restricting probabilities on both sides to the event $\{\tau>0\}$, which is because in this case, the third line of the last displayed equation in the above proof is further rewritten as

$$
\begin{aligned}
& 2 K_{0}\left(\sqrt{\mu^{2}+\xi^{2}}\right) f(0) \mathbb{P}(\tau=0) \\
& +\int_{0}^{\infty} \frac{d v}{v} \exp \left(-\frac{1}{2 v}\right) \exp \left(-\frac{\mu^{2}}{2} v\right) \mathbb{E}\left[f\left(Z_{\tau}\right) \exp \left\{-\frac{\xi^{2}}{2}\left(e^{2 B_{\tau}} v+A_{\tau}\right)\right\} ; \tau>0\right]
\end{aligned}
$$

by (2.10) if $f$ is a bounded measurable function on $[0, \infty)$.

In view of Propositions 2.2, the above Proposition 3.4 may also be seen as a consequence of the relation

$$
\begin{aligned}
& \frac{1}{v} \exp \left(-\frac{1}{2 v}\right) \frac{\mathbb{P}\left(e^{2 z_{\mu}} v+e^{z_{\mu}} / \mu \in d u\right)}{d u} \\
& =\frac{1}{u} \exp \left(-\frac{1}{2 u}\right) \frac{\mathbb{P}\left(e^{2 z_{\mu}} u+e^{z_{\mu}} / \mu \in d v\right)}{d v}, \quad u, v>0
\end{aligned}
$$


for every $\mu>0$, which follows readily from Proposition 2.3 by the same reasoning as in the above proof. A simple computation shows that the left-hand side, and hence the right-hand side as well, of (3.23) admits the representation

$$
\frac{1}{4 K_{0}(\mu)} \frac{1}{u v}\left(1+\frac{1}{\sqrt{1+4 \mu^{2} u v}}\right) \exp \left\{-\frac{u+v}{4 u v}\left(\sqrt{1+4 \mu^{2} u v}+1\right)\right\},
$$

which is indeed symmetric with respect to $u$ and $v$. From this representation together with (2.26), it follows that when $\tau=t>0$, both sides of (3.20) admit the density function

$$
\frac{1}{2 u v w}\left(1+\frac{1}{\sqrt{1+4 u v / w^{2}}}\right) \exp \left\{-\frac{u+v}{4 u v}\left(\sqrt{1+4 u v / w^{2}}+1\right)\right\} \Theta_{1 / w}(t)
$$

with respect to the Lebesgue measure $d u d v d w$ on $(0, \infty)^{3}$.

Remark 3.4. For every $v>0$, the Laplace transform of the law of $e^{2 B_{\tau}} v+A_{\tau}$ may be expressed as follows: for any $\xi \in \mathbb{R}$,

$$
\begin{aligned}
& \mathbb{E}\left[\exp \left\{-\frac{\xi^{2}}{2}\left(e^{2 B_{\tau}} v+A_{\tau}\right)\right\}\right] \\
& =\mathbb{E}\left[\cos \left\{\xi \sinh \left(\operatorname{Argsh} \hat{\beta}(v)+B_{\tau}\right)\right\}\right] \\
& =\int_{\mathbb{R}} \frac{d y}{\sqrt{2 \pi v}} \cosh y \exp \left(-\frac{\sinh ^{2} y}{2 v}\right) \mathbb{E}\left[\cos \left\{\xi \sinh \left(y+B_{\tau}\right)\right\}\right],
\end{aligned}
$$

where in the second line, $\hat{\beta}$ is a Brownian motion independent of $B$, and the third line follows by a simple computation. To see the first equality, it suffices to note that by (1.9),

$$
\begin{aligned}
\sinh \left(\operatorname{Argsh} \hat{\beta}(v)+B_{\tau}\right) & \stackrel{(d)}{=} e^{B_{\tau}} \hat{\beta}(v)+\beta\left(A_{\tau}\right) \\
& \stackrel{(d)}{=} \hat{\beta}\left(e^{2 B_{\tau}} v+A_{\tau}\right)
\end{aligned}
$$

where in the second line, we still denote by $\hat{\beta}$ a Brownian motion independent of $B$.

\subsection{Analytic applications of Proposition 1.1 and Lemma 2.1}

In this subsection, we give one analytic application of Proposition 1.1, as well as other two analytic applications of Lemma 2.1, one of which uses the case $\xi=0$, and the other of which uses the case $\lambda=0$ as has already been applied in Subsection 2.3.

We start with derivation of the following integral representation for the Macdonald function $K_{\nu}$ with $\nu>-1 / 2$, by means of Proposition 1.1.

Proposition 3.5. If $\nu>-1 / 2$, then it holds that

$$
K_{\nu}(z)=\frac{\sqrt{\pi} z^{\nu}}{2^{\nu} \Gamma(\nu+1 / 2)} \int_{0}^{\infty} d x e^{-z \cosh x} \sinh ^{2 \nu} x, \quad z>0
$$


For the representation (3.24), see, e.g., [17, p. 140, Problem 6].

Proof of Proposition 3.5. By symmetry and by Proposition 1.1, the integral in the righthand side of (3.24) is equal to

$$
\begin{aligned}
& \frac{1}{2} \int_{\mathbb{R}} d x e^{-z \cosh x}|\sinh x|^{2 \nu} \\
& =\frac{1}{2} \int_{0}^{\infty} \frac{d v}{v} \exp \left(-\frac{1}{2 v}\right) \exp \left(-\frac{z^{2}}{2} v\right) \mathbb{E}\left[|\beta(v)|^{2 \nu}\right] .
\end{aligned}
$$

Note that when $\nu>-1 / 2$, we have

$$
\begin{aligned}
\mathbb{E}\left[|\beta(v)|^{2 \nu}\right] & =v^{\nu} \mathbb{E}\left[|\beta(1)|^{2 \nu}\right] \\
& =v^{\nu} \times \frac{2^{\nu}}{\sqrt{\pi}} \Gamma(\nu+1 / 2)
\end{aligned}
$$

for every $v>0$, where the first line is due to the scaling property of Brownian motion and the second follows readily from the fact that $|\beta(1)|^{2} \stackrel{(d)}{=} 2 \gamma_{1 / 2}$. By plugging the last expression into (3.25), we see that the right-hand side of the claimed formula (3.24) is written as

$$
\frac{1}{2} z^{\nu} \int_{0}^{\infty} d v v^{\nu-1} \exp \left\{-\frac{1}{2}\left(\frac{1}{v}+z^{2} v\right)\right\}
$$

which is equal to $K_{\nu}(z)$ in view of (2.8) as well as of (2.7).

We turn to the two applications of Lemma 2.1. The first one concerns an integral representation of products of two Macdonald functions, which is found, e.g., in [17, p. 140, Problem 7].

Proposition 3.6. For every $\nu \in \mathbb{R}$, it hold that

$$
K_{\nu}(z) K_{\nu}(w)=\frac{1}{2} \int_{0}^{\infty} \frac{d v}{v} \exp \left(-\frac{1}{2 v}\right) \exp \left(-\frac{z^{2}+w^{2}}{2} v\right) K_{\nu}(z w v), \quad z, w>0 .
$$

Proof. By the integral representation (2.4) of $K_{\nu}$, we plug the expression

$$
K_{\nu}(z w v)=\frac{1}{2} \int_{\mathbb{R}} d x e^{-z w v \cosh x} e^{-\nu x}
$$

into the right-hand side of (3.26). Then by Fubini's theorem, it is rewritten as

$$
\frac{1}{4} \int_{\mathbb{R}} d x e^{-\nu x} \int_{0}^{\infty} \frac{d v}{v} \exp \left(-\frac{1}{2 v}\right) \exp \left(-\frac{z^{2}+w^{2}}{2} v\right) e^{-z w v \cosh x} .
$$

Applying Lemma 2.1 with $\mu=z, \lambda=w$ and $\xi=0$, we see that the integral with respect to $v$ above is equal to

$$
\int_{\mathbb{R}} d y e^{-z \cosh y} e^{-w \cosh (x+y)}
$$


Therefore by using Fubini's theorem again, the expression (3.27) is further rewritten as

$$
\frac{1}{2} \int_{\mathbb{R}} d y e^{-z \cosh y} e^{\nu y} \times \frac{1}{2} \int_{\mathbb{R}} d x e^{-w \cosh (x+y)} e^{-\nu(x+y)},
$$

which agrees with the left-hand side of (3.26) in view of (2.4).

The second application of Lemma 2.1 deals with an integral representation for the density function of a given symmetric random variable.

Proposition 3.7. Let $X$ be a symmetric random variable and suppose that it satisfies

$$
\int_{\mathbb{R}} d x e^{-\mu \cosh x} \int_{\mathbb{R}} d \xi|\mathbb{E}[\cos \{\xi \sinh (x+X)\}]|<\infty \quad \text { for any } \mu>0 .
$$

Then $X$ admits the density function $\varphi$ given by

$$
\varphi(x)=\frac{1}{2 \pi} \int_{\mathbb{R}} d \xi \mathbb{E}[\cos \{\xi \sinh (x+X)\}], \quad x \in \mathbb{R} .
$$

Proof. What we are going to use is Lemma 2.1 with $\lambda=0$, namely the integral identity (2.33). We substitute $x$ by $X$ and take the expectation on both sides to get

$$
\begin{aligned}
& \int_{\mathbb{R}} d y e^{-\mu \cosh y} \mathbb{E}[\cos \{\xi \sinh (y+X)\}] \\
& =\mathbb{E}\left[\int_{0}^{\infty} \frac{d v}{v} \exp \left(-\frac{1}{2 v}\right) \exp \left(-\frac{\mu^{2}+\xi^{2}}{2} v\right) e^{i \mu \xi v \sinh X}\right],
\end{aligned}
$$

where on the left-hand side, we used Fubini's theorem and the fact that the mapping $\mathbb{R} \ni y \mapsto \mathbb{E}[\sin \{\xi \sinh (y+X)\}]$ is an odd function due to symmetry of $X$. We integrate both sides of the above equality with respect to $\xi$ over $\mathbb{R}$. Then by the condition (3.28) and by Fubini's theorem, the left-hand side of (3.30) turns into

$$
2 \pi \int_{\mathbb{R}} d y e^{-\mu \cosh y} \varphi(y)
$$

with function $\varphi$ given in (3.29). On the other hand, as for the right-hand side of (3.30), by observing that

$$
\int_{\mathbb{R}} d \xi \exp \left(-\frac{\xi^{2}}{2} v\right) e^{i \mu \xi v \sinh X}=\sqrt{\frac{2 \pi}{v}} \exp \left(-\frac{\mu^{2} \sinh ^{2} X}{2} v\right),
$$

Fubini's theorem allows us to compute

$$
\begin{aligned}
& 2 \pi \mathbb{E}\left[\int_{0}^{\infty} \frac{d v}{\sqrt{2 \pi v^{3}}} \exp \left(-\frac{1}{2 v}\right) \exp \left(-\frac{\mu^{2} \cosh ^{2} X}{2} v\right)\right] \\
& =2 \pi \mathbb{E}\left[\exp \left\{-\frac{\mu^{2} \cosh ^{2} X}{2} \tau_{1}(\hat{B})\right\}\right] \\
& =2 \pi \mathbb{E}[\exp (-\mu \cosh X)]
\end{aligned}
$$

thanks to (2.1) and (1.16), where in the second line, $\hat{B}$ denotes a Brownian motion independent of $X$. Since the last expression agrees with (3.31) for any $\mu>0$, we obtain the conclusion owing to symmetry of $X$. 
We may compare the above proposition with the well-known fact (see, e.g., [10, Theorem 3.3.5]) that if a generic random variable $X$ satisfies the condition $\int_{\mathbb{R}} d \xi\left|\mathbb{E}\left[e^{i \xi X}\right]\right|<$ $\infty$, then it admits the density function

$$
\varphi(x)=\frac{1}{2 \pi} \int_{\mathbb{R}} d \xi e^{-i x \xi} \mathbb{E}\left[e^{i \xi X}\right], \quad x \in \mathbb{R} .
$$

If we apply this general fact to a symmetric $X$, then the integral representation (3.32) may be written as

$$
\begin{aligned}
\varphi(x) & =\frac{1}{2 \pi} \int_{\mathbb{R}} d \xi \cos (x \xi) \mathbb{E}[\cos (\xi X)] \\
& =\frac{1}{2 \pi} \int_{\mathbb{R}} d \xi \mathbb{E}[\cos \{\xi(x+X)\}], \quad x \in \mathbb{R} .
\end{aligned}
$$

Proposition 3.7 asserts that for symmetric random variables $X$ of a certain class, two expressions (3.29) and (3.33) agree.

We give examples of symmetric random variables satisfying the condition (3.28).

Example 3.1. (1) Brownian motion at fixed time $t>0$ fulfills (3.28). To verify it, note that by the identity (1.2),

$$
\begin{aligned}
\left|\mathbb{E}\left[\cos \left\{\xi \sinh \left(x+B_{t}\right)\right\}\right]\right| & =\left|\mathbb{E}\left[\cos \left(\xi e^{B_{t}} \sinh x\right) \exp \left(-\frac{\xi^{2}}{2} A_{t}\right)\right]\right| \\
& \leq \mathbb{E}\left[\exp \left(-\frac{\xi^{2}}{2} A_{t}\right)\right]
\end{aligned}
$$

for any $x, \xi \in \mathbb{R}$. Therefore the double integral in the condition (3.28) is dominated by

$$
\begin{aligned}
& \int_{\mathbb{R}} d x e^{-\mu \cosh x} \times \int_{\mathbb{R}} d \xi \mathbb{E}\left[\exp \left(-\frac{\xi^{2}}{2} A_{t}\right)\right] \\
& =2 \sqrt{2 \pi} K_{0}(\mu) \mathbb{E}\left[\frac{1}{\sqrt{A_{t}}}\right],
\end{aligned}
$$

which is finite by the fact that

$$
\mathbb{E}\left[\frac{1}{\sqrt{A_{t}}}\right]=\frac{1}{\sqrt{t}}
$$

for any $t>0$. The above fact may be seen from the identity (1.2) in such a way that two density functions in $y \in \mathbb{R}$ :

$$
\frac{\mathbb{P}\left(e^{B_{t}} \sinh x+\beta\left(A_{t}\right) \in d y\right)}{d y}=\mathbb{E}\left[\frac{1}{\sqrt{2 \pi A_{t}}} \exp \left\{-\frac{\left(y-e^{B_{t}} \sinh x\right)^{2}}{2 A_{t}}\right\}\right]
$$

and

$$
\frac{\mathbb{P}\left(\sinh \left(x+B_{t}\right) \in d y\right)}{d y}=\frac{1}{\sqrt{2 \pi t}} \frac{1}{\sqrt{1+y^{2}}} \exp \left\{-\frac{(\operatorname{Argsh} y-x)^{2}}{2 t}\right\}
$$


agree for every $x \in \mathbb{R}$ and evaluating them at $x=y=0$ leads to the claimed equality. It should also be noted that since $X=B_{t}$ fulfills $\int_{\mathbb{R}} d \xi\left|\mathbb{E}\left[e^{i \xi X}\right]\right|<\infty$, the expression (3.33) is valid as well; in fact, a direct computation shows that

$$
\frac{1}{2 \pi} \int_{\mathbb{R}} d \xi \cos (x \xi) \exp \left(-\frac{t}{2} \xi^{2}\right)=\frac{1}{\sqrt{2 \pi t}} \exp \left(-\frac{x^{2}}{2 t}\right), \quad x \in \mathbb{R}
$$

(2) From the above argument in (1), it is now clear that Brownian motion evaluated at an independent random time $T$ satisfying

$$
\mathbb{E}\left[\frac{1}{\sqrt{T}}\right]<\infty
$$

fulfills (3.28). A typical example of such situations is given by a symmetric Cauchy variable $a C$ for every $a \neq 0$, because it holds that $a C \stackrel{(d)}{=} \beta\left(\tau_{a}(\hat{B})\right)$ as seen in (2.31) and that by (2.1), $\tau_{a}(\hat{B})$ satisfies

$$
\begin{aligned}
\mathbb{E}\left[\frac{1}{\sqrt{\tau_{a}(\hat{B})}}\right] & =\frac{|a|}{\sqrt{2 \pi}} \int_{0}^{\infty} \frac{d v}{v^{2}} \exp \left(-\frac{a^{2}}{2 v}\right) \\
& <\infty
\end{aligned}
$$

Among other examples, we have $\left(1 / Z_{t}\right) \sinh B_{t}$ and $\left(1 / \sqrt{Z_{t}}\right) \sinh B_{t}$ for each $t>0$, as is deduced from identities (1.12) and (1.13).

(3) For each $u>0$, the random variable $z_{u}$ fulfills (3.28). (Here we replace by $u$ the exponent $\mu$ in the definition (2.3) of $z_{\mu}$ to avoid confusion.) More generally, if a symmetric random variable $X$ is such that

$$
\mathbb{P}(X \in d x)=\int_{0}^{\infty} m(d u) \mathbb{P}\left(z_{u} \in d x\right), \quad x \in \mathbb{R},
$$

for some probability measure $m$ on $(0, \infty)$ satisfying

$$
\int_{0}^{\infty} m(d u) \sqrt{u} \mathbb{E}\left[e^{-z_{u} / 2}\right]<\infty,
$$

then $X$ fulfills (3.28). Examples in (1) and (2) above may be seen as consequences of this general statement; indeed, if we take $m(d u)=\mathbb{P}\left(1 / Z_{t} \in d u\right)$ for $t>0$, then by Proposition 2.2,

$$
\int_{0}^{\infty} m(d u) \sqrt{u} \mathbb{E}\left[e^{-z_{u} / 2}\right]=\mathbb{E}\left[\frac{1}{\sqrt{A_{t}}}\right]
$$

In order to draw the above condition (3.36), we use in place of (1.2) the identity

$$
\sinh \left(x+z_{u}\right) \stackrel{(d)}{=} e^{z_{u}} \sinh x+\beta\left(e^{z_{u}} / u\right)
$$


adopted from Proposition 2.3 and argue along the same lines as in (1) to see that

$$
\begin{aligned}
\int_{\mathbb{R}} d \xi\left|\mathbb{E}\left[\cos \left\{\xi \sinh \left(x+z_{u}\right)\right\}\right]\right| & \leq \int_{\mathbb{R}} d \xi \mathbb{E}\left[\exp \left(-\frac{\xi^{2}}{2} \frac{e^{z_{u}}}{u}\right)\right] \\
& =\sqrt{2 \pi u} \mathbb{E}\left[e^{-z_{u} / 2}\right]
\end{aligned}
$$

which leads to (3.36). If we note the identities

$$
\begin{aligned}
\mathbb{E}\left[e^{-z_{u} / 2}\right] & =\frac{K_{1 / 2}(u)}{K_{0}(u)} \\
& =\sqrt{\frac{\pi}{2 u}} \frac{e^{-u}}{K_{0}(u)},
\end{aligned}
$$

then the condition (3.36) is restated as $\int_{0}^{\infty} m(d u) e^{-u} / K_{0}(u)<\infty$. In the last displayed equations, we used (2.4) for the first line and the explicit expression of $K_{1 / 2}$ (see, e.g., [17, Equation (5.8.5)]) for the second.

As already seen partly in (1) of the above example, in the case $X=B_{t}$ for $t>0$, the right-hand side of (3.29) is expressed, by the identity (1.2), as

$$
\frac{1}{2 \pi} \int_{\mathbb{R}} d \xi \mathbb{E}\left[\cos \left(\xi e^{B_{t}} \sinh x\right) \exp \left(-\frac{\xi^{2}}{2} A_{t}\right)\right]
$$

By Fubini's theorem, this expression is equal to

$$
\frac{1}{2 \pi} \mathbb{E}\left[\int_{\mathbb{R}} d \xi \cos \left(\xi e^{B_{t}} \sinh x\right) \exp \left(-\frac{\xi^{2}}{2} A_{t}\right)\right]=\mathbb{E}\left[\frac{1}{\sqrt{2 \pi A_{t}}} \exp \left(-\frac{e^{2 B_{t}} \sinh ^{2} x}{2 A_{t}}\right)\right],
$$

and hence Proposition 3.7 entails the relation

$$
\mathbb{E}\left[\frac{1}{\sqrt{2 \pi A_{t}}} \exp \left(-\frac{e^{2 B_{t}} \sinh ^{2} x}{2 A_{t}}\right)\right]=\frac{1}{\sqrt{2 \pi t}} \exp \left(-\frac{x^{2}}{2 t}\right)
$$

for every $t>0$ and $x \in \mathbb{R}$. The above probabilistic representation for the Gaussian kernel is also obtained by evaluating (3.34) and (3.35) at $y=0$. Moreover, thanks to the formula (2.22), the left-hand side of (3.37) is calculated as

$$
\begin{aligned}
& \int_{0}^{\infty} \frac{d v}{v} \int_{0}^{\infty} \frac{d u}{u} \exp \left(-\frac{1+u^{2}}{2 v}\right) \Theta_{u / v}(t) \frac{1}{\sqrt{2 \pi v}} \exp \left(-\frac{u^{2} \sinh ^{2} x}{2 v}\right) \\
& =\int_{0}^{\infty} \frac{d u}{u} \Theta_{u}(t) \int_{0}^{\infty} \frac{d v}{\sqrt{2 \pi v^{3}}} \exp \left(-\frac{1}{2 v}\right) \exp \left(-\frac{u^{2} \cosh ^{2} x}{2} v\right) \\
& =\int_{0}^{\infty} \frac{d u}{u} \Theta_{u}(t) e^{-u \cosh x},
\end{aligned}
$$

where for the second line, we changed the variable $u$ into $v u$ and used Fubini's theorem, and for the third line, we used (2.1) and (1.16). Therefore in addition to (2.23), we have 
another characterization of the function $\Theta_{r}(t), r>0, t>0$, in terms of the Laplace transform in variable $r$ :

$$
\int_{0}^{\infty} \frac{d r}{r} \Theta_{r}(t) e^{-r \cosh x}=\frac{1}{\sqrt{2 \pi t}} \exp \left(-\frac{x^{2}}{2 t}\right), \quad t>0, x \in \mathbb{R} .
$$

We remark that this relation is stated in [20, Proposition 4.5 (i)] and, as observed in [23, Proposition 4.2], also follows by simply integrating both sides of (2.22) with respect to $v$ over $(0, \infty)$. As seen above, the relation (3.38) explains the coincidence of the two expressions (3.34) and (3.35) in the case $y=0$. It is not hard to see similarly that their coincidence in the case $y \neq 0$ is also reduced to the above relation; in fact, by using (2.22), the expectation in (3.34) is calculated as

$$
\frac{1}{\sqrt{1+y^{2}}} \int_{0}^{\infty} \frac{d u}{u} \Theta_{u}(t) \exp \{-u \cosh (\operatorname{Argsh} y-x)\},
$$

which agrees with (3.35) thanks to the relation (3.38). In Subsection A.3 of the appendix, we derive from (3.38) the integral representation (2.24) for $\Theta_{r}(t)$.

\section{Concluding Remarks}

In this paper we have shown, with the help of Proposition 2.2 due to Matsumoto and Yor, that Bougerol's identity (1.1) as well as its extensions in Theorems 1.1 and 1.2 are obtained from relevant properties of random variables $z_{\mu}, \mu>0$, defined in (2.3). In particular, we have shown that for every fixed $t>0$ and $x \in \mathbb{R}$, there takes place the coincidence of joint laws

$$
\left(e^{B_{t}} \sinh x+\beta\left(A_{t}\right), e^{-B_{t}} A_{t}\right) \stackrel{(d)}{=}\left(\sinh \left(x+B_{t}\right), e^{-B_{t}} A_{t}\right) .
$$

In view of an expression (1.6) of the process $Y^{x}$ defined by (1.3), the left-hand side of (4.1) is identical in law with

$$
\left(Y_{t}^{x}, e^{-B_{t}} A_{t}\right)
$$

Indeed, we have

$$
\begin{aligned}
\left(Y_{t}^{x}, e^{-B_{t}} A_{t}\right) \stackrel{(d)}{=}\left(e^{B_{t}} \sinh x+e^{B_{t}} \beta\left(e^{-2 B_{t}} A_{t}\right), e^{-B_{t}} A_{t}\right) \\
\stackrel{(d)}{=}\left(e^{B_{t}} \sinh x+\beta\left(A_{t}\right), e^{-B_{t}} A_{t}\right)
\end{aligned}
$$

where the first line follows from the relation (11.8) and the second is due to the scaling property of Brownian motion as was seen in (1.7). Recall that the process $\beta^{x}$ defined in (1.4) is a Brownian motion. Then, since the right-hand side of (4.1) is identical in law with

$$
\left(Y_{t}^{x}, e^{-\beta_{t}^{x}} \int_{0}^{t} e^{2 \beta_{s}^{x}} d s\right)
$$


in view of the other expression (1.5) of $Y^{x}$, we may rephrase (4.1) as

$$
\left(Y_{t}^{x}, e^{-B_{t}} \int_{0}^{t} e^{2 B_{s}} d s\right) \stackrel{(d)}{=}\left(Y_{t}^{x}, e^{-\beta_{t}^{x}} \int_{0}^{t} e^{2 \beta_{s}^{x}} d s\right) .
$$

It would be interesting to give a direct explanation to the identity (4.2) by means of Itô's formula and SDEs, which we think should lead us to a deeper understanding, such as another proof, of the explicit formula (2.22) for the joint law of $e^{B_{t}}$ and $A_{t}$.

\section{Appendix}

We append some explorations as to the bivariate function $\Theta_{r}(t), r>0, t>0$, that is characterized by (2.23) as well as by (3.38), and admits the integral representation (2.24).

\section{A.1 An integral equation for $\Theta_{r}(t)$}

In the first part of the appendix, we derive an integral equation satisfied by $\Theta_{r}(t)$. To begin with, we note that the integral representation (2.4) for the Macdonald function $K_{\nu}$ is valid if $\nu$ is in $\mathbb{C}$, the complex plane, and that when $\nu=i \xi, \xi \in \mathbb{R}$, it reads

$$
K_{i \xi}(z)=\frac{1}{2} \int_{\mathbb{R}} d x e^{-z \cosh x} \cos (\xi x), \quad z>0 .
$$

Proposition A.1. For every $r>0$ and $t>0$, it holds that

$$
\Theta_{r}(t)=\frac{r}{t} \exp \left(\frac{\pi^{2}}{2 t}\right) \int_{0}^{\infty} \frac{d u}{u(u+r)} K_{\pi i / t}(u+r) \Theta_{u}(t) .
$$

Proof. In the integral representation (2.24) for $\Theta_{r}(t)$, the integrand is a symmetric function in $y \in \mathbb{R}$, and hence we may represent $\Theta_{r}(t)$ as

$$
\Theta_{r}(t)=\frac{r}{2 \pi} \exp \left(\frac{\pi^{2}}{2 t}\right) \mathbb{E}\left[\exp \left(-r \cosh B_{t}\right) \sinh B_{t} \sin \left(\frac{\pi}{t} B_{t}\right)\right] .
$$

By noting the relation (3.38) and using Fubini's theorem, the expectation on the righthand side may be written as

$$
\int_{0}^{\infty} \frac{d u}{u} \Theta_{u}(t) \int_{\mathbb{R}} d x \exp \{-(u+r) \cosh x\} \sinh x \sin \left(\frac{\pi}{t} x\right) .
$$

By applying the integration by parts formula, the integral with respect to $x$ in the last expression is calculated as

$$
\begin{aligned}
& {\left[-\frac{1}{u+r} \exp \{-(u+r) \cosh x\} \sin \left(\frac{\pi}{t} x\right)\right]_{x=-\infty}^{\infty}} \\
& +\frac{\pi}{t(u+r)} \int_{\mathbb{R}} d x \exp \{-(u+r) \cosh x\} \cos \left(\frac{\pi}{t} x\right) \\
& =\frac{2 \pi}{t(u+r)} K_{\pi i / t}(u+r)
\end{aligned}
$$


thanks to (A.1). Hence the expectation on the right-hand side of (A.3) is equal to

$$
\frac{2 \pi}{t} \int_{0}^{\infty} \frac{d u}{u(u+r)} \Theta_{u}(t) K_{\pi i / t}(u+r),
$$

which proves the relation $(\underline{A .2}$ ) as desired.

\section{A.2 Derivation of Proposition 3.3 from (3.38)}

Recall from Proposition 3.3 the following identities between expectations relative to Brownian motion $B$ : for every $\lambda \geq 0$ and $\xi \in \mathbb{R}$,

$$
\begin{aligned}
& \mathbb{E}\left[\exp \left(-\lambda e^{B_{t}}-\frac{\xi^{2}}{2} A_{t}\right)\right] \\
& = \begin{cases}\mathbb{E}\left[\exp \left(-\lambda \cosh B_{t}\right) \cos \left(\sqrt{\xi^{2}-\lambda^{2}} \sinh B_{t}\right)\right] & \text { if } \lambda \leq|\xi|, \\
\mathbb{E}\left[\exp \left(-\lambda \cosh B_{t}\right) \cosh \left(\sqrt{\lambda^{2}-\xi^{2}} \sinh B_{t}\right)\right] & \text { if } \lambda \geq|\xi| .\end{cases}
\end{aligned}
$$

As was seen in (3.19), the left-hand side admits the representation

$$
2 \int_{0}^{\infty} \frac{d r}{r} K_{0}\left(\sqrt{(r+\lambda)^{2}+\xi^{2}-\lambda^{2}}\right) \Theta_{r}(t)
$$

which was obtained by calculating the expectation

$$
\mathbb{E}\left[\exp \left(-\lambda e^{z_{\mu}}-\frac{\xi^{2}}{2} \frac{e^{z_{\mu}}}{\mu}\right)\right]
$$

for each $\mu>0$. In this part of the appendix, based on the relation (3.38) and Proposition 1.1, we derive the representation (A.5) from expressions on the right-hand side of (A.4), which we think would provide us with a better understanding of the identities (A.4).

We treat the case $\lambda \leq|\xi|$ first. Note that by (3.38) and Fubini's theorem, the right-hand side of (A.4) in this case is rewritten as

$$
\int_{0}^{\infty} \frac{d r}{r} \Theta_{r}(t) \int_{\mathbb{R}} d x \exp \{-(r+\lambda) \cosh x\} \cos \left(\sqrt{\xi^{2}-\lambda^{2}} \sinh x\right)
$$

By Proposition 1.1, the integral with respect to $x$ in the expression (A.6) is equal to

$$
\int_{0}^{\infty} \frac{d v}{v} \exp \left(-\frac{1}{2 v}\right) \exp \left\{-\frac{(r+\lambda)^{2}}{2} v\right\} \mathbb{E}\left[\cos \left\{\sqrt{\xi^{2}-\lambda^{2}} \beta(v)\right\}\right] .
$$

Since there holds the equality

$$
\mathbb{E}\left[\cos \left\{\sqrt{\xi^{2}-\lambda^{2}} \beta(v)\right\}\right]=\exp \left(-\frac{\xi^{2}-\lambda^{2}}{2} v\right),
$$


the above integral with respect to $v$ is equal to

$$
2 K_{0}\left(\sqrt{(r+\lambda)^{2}+\xi^{2}-\lambda^{2}}\right)
$$

by (2.10), which proves that (A.6) agrees with (A.5).

By replacing $\cos \left(\sqrt{\xi^{2}-\lambda^{2}} \sinh x\right)$ in (A.6) by $\cosh \left(\sqrt{\lambda^{2}-\xi^{2}} \sinh x\right)$, the case $\lambda \geq$ $|\xi|$ is treated in a similar way owing to the fact that

$$
\begin{aligned}
\mathbb{E}\left[\cosh \left\{\sqrt{\lambda^{2}-\xi^{2}} \beta(v)\right\}\right] & =\mathbb{E}\left[e^{\sqrt{\lambda^{2}-\xi^{2}} \beta(v)}\right] \\
& =\exp \left(\frac{\lambda^{2}-\xi^{2}}{2} v\right)
\end{aligned}
$$

where the first equality is due to symmetry of Brownian motion.

\section{A.3 Explanation of (2.24) via (3.38)}

In [29] (see also [23, Appendix A]), Yor obtained the integral representation (2.24) for $\Theta_{r}(t)$ by inverting its Laplace transform (2.23) taken with respect to variable $t$. In the last part of this appendix, we explain (2.24) via (3.38), the Laplace transform with respect to variable $r$.

We fix $t>0$ below. By the representation (A.3) and Fubini's theorem, the left-hand side of the relation (3.38) may be written as

$$
\frac{1}{2 \pi} \exp \left(\frac{\pi^{2}}{2 t}\right) \mathbb{E}\left[\frac{\sinh B_{t} \sin \left(\pi B_{t} / t\right)}{\cosh B_{t}+\cosh x}\right], \quad x \in \mathbb{R} .
$$

Hence if we take the Fourier transform on both sides of (3.38), it reads

$$
\frac{1}{2 \pi} \exp \left(\frac{\pi^{2}}{2 t}\right) \int_{\mathbb{R}} d x \cos (\xi x) \mathbb{E}\left[\frac{\sinh B_{t} \sin \left(\pi B_{t} / t\right)}{\cosh B_{t}+\cosh x}\right]=\exp \left(-\frac{\xi^{2}}{2} t\right), \quad \xi \in \mathbb{R} .
$$

Therefore thanks to the injectivity of Fourier and Laplace transforms, in order to verify the representation (2.24), it suffices to show that for any $\xi \in \mathbb{R}$,

$$
\int_{\mathbb{R}} d x \cos (\xi x) \mathbb{E}\left[\frac{\sinh B_{t} \sin \left(\pi B_{t} / t\right)}{\cosh B_{t}+\cosh x}\right]=2 \pi \exp \left(-\frac{\pi^{2}}{2 t}-\frac{\xi^{2}}{2} t\right) .
$$

Let $\xi \neq 0$ for a while and note the fact that for any $b \in \mathbb{R}$ with $b \neq 0$,

$$
\int_{\mathbb{R}} d x \frac{\cos (\xi x)}{\cosh b+\cosh x}=\frac{2 \pi \sin (\xi b)}{\sinh (\pi \xi) \sinh b}
$$

Indeed, it is known (cf. [28, Subsection 13.21, Equation (9)]) that

$$
\int_{0}^{\infty} d u e^{-u \cosh b} K_{i \xi}(u)=\frac{\pi \sin (\xi b)}{\sinh (\pi \xi) \sinh b}
$$


into the left-hand side of which we plug the integral representation (A.1) of $K_{i \xi}$ to obtain by Fubini's theorem,

$$
\begin{aligned}
\int_{0}^{\infty} d u e^{-u \cosh b} K_{i \xi}(u) & =\frac{1}{2} \int_{\mathbb{R}} d x \cos (\xi x) \int_{0}^{\infty} d u e^{-u(\cosh b+\cosh x)} \\
& =\frac{1}{2} \int_{\mathbb{R}} d x \frac{\cos (\xi x)}{\cosh b+\cosh x}
\end{aligned}
$$

Using the above fact (A.8) and Fubini's theorem, we then see that the left-hand side of (A.7) is equal to

$$
\begin{aligned}
& \frac{2 \pi}{\sinh (\pi \xi)} \mathbb{E}\left[\sinh B_{t} \sin \left(\frac{\pi}{t} B_{t}\right) \frac{\sin \left(\xi B_{t}\right)}{\sinh B_{t}} ; B_{t} \neq 0\right] \\
& =\frac{\pi}{\sinh (\pi \xi)} \mathbb{E}\left[\cos \left\{\left(\frac{\pi}{t}-\xi\right) B_{t}\right\}-\cos \left\{\left(\frac{\pi}{t}+\xi\right) B_{t}\right\}\right] \\
& =\frac{\pi}{\sinh (\pi \xi)}\left[\exp \left\{-\frac{1}{2}\left(\frac{\pi}{t}-\xi\right)^{2} t\right\}-\exp \left\{-\frac{1}{2}\left(\frac{\pi}{t}+\xi\right)^{2} t\right\}\right]
\end{aligned}
$$

which agrees with the right-hand side of (A.7). The validity of (A.7) in the case $\xi=0$ is now clear since both sides of (A.7) are continuous functions in $\xi$.

If we consider the meromorphic function

$$
f(z)=\frac{\cos (\xi z)}{\cosh b+\cosh z}
$$

on $\mathbb{C}$, then by noting the fact that the poles $w$ of $f$ each of whose imaginary part $\operatorname{Im} w$ satisfies $0<\operatorname{Im} w<2 \pi$ are $\pm b+\pi i$, the above formula (A.8) may be verified by standard residue calculus along a rectangular contour circling $\pm b+\pi i$ and having its two sides on the two lines $\operatorname{Im} z=0$ and $\operatorname{Im} z=2 \pi$. We also note that the formula (A.9) may be seen from the following particular case of the Hankel-Lipschitz formulae (cf. [12, p. 703], [28, Subsection 13.21]): for every $\nu \in \mathbb{C}$ whose real part is strictly greater than -1 ,

$$
\int_{0}^{\infty} d u e^{-u \cosh b} I_{\nu}(u)=\frac{e^{-\nu b}}{\sinh b}, \quad b>0,
$$

together with the definition of $K_{\nu}$ for a noninteger $\nu$ (see [17, Section 5.7]):

$$
K_{\nu}(b)=\frac{\pi}{2} \frac{I_{-\nu}(b)-I_{\nu}(b)}{\sin (\pi \nu)} .
$$

Remark A.1. The same computation as in the above verification of the formula (A.7) also proves that when $t>0$ and $\xi \neq 0$,

$$
\int_{\mathbb{R}} d x \cos (\xi x) \mathbb{E}\left[\frac{\sinh B_{t} \sin \left(\alpha B_{t} / t\right)}{\cosh B_{t}+\cosh x}\right]=2 \pi \exp \left(-\frac{\alpha^{2}}{2 t}-\frac{\xi^{2}}{2} t\right) \frac{\sinh (\alpha \xi)}{\sinh (\pi \xi)}
$$


for any $\alpha \in \mathbb{R}$. Fourier inversion of the right-hand side gives us several formulae for expectations as in the left-hand side of (A.10) for different values of $\alpha$. When $\alpha=2 \pi$ for example, since it is readily seen that for any $\xi \in \mathbb{R}$,

$$
\int_{\mathbb{R}} d x \cos (\xi x) \frac{1}{\sqrt{2 \pi t}} \exp \left(-\frac{x^{2}}{2 t}\right) \cos \left(\frac{\pi}{t} x\right)=\exp \left(-\frac{\pi^{2}}{2 t}-\frac{\xi^{2}}{2} t\right) \cosh (\pi \xi),
$$

we have

$$
\mathbb{E}\left[\frac{\sinh B_{t} \sin \left(2 \pi B_{t} / t\right)}{\cosh B_{t}+\cosh x}\right]=2 \sqrt{\frac{2 \pi}{t}} \exp \left(-\frac{3 \pi^{2}}{2 t}-\frac{x^{2}}{2 t}\right) \cos \left(\frac{\pi}{t} x\right), \quad x \in \mathbb{R},
$$

which, after replacing $t$ and $x$ by $4 t$ and $2 x$, respectively, agrees with [22, Lemma 3.1]. In the case $\alpha=\pi / 2$, the right-hand side of (A.10) becomes

$$
\pi \exp \left(-\frac{\pi^{2}}{8 t}-\frac{\xi^{2}}{2} t\right) \frac{1}{\cosh (\pi \xi / 2)}
$$

and noting the fact that $1 / \cosh (\pi \xi / 2), \xi \in \mathbb{R}$, is the characteristic function of $\log |C|$ whose probability density is given by $1 /(\pi \cosh x), x \in \mathbb{R}$ (cf. [25, Chapter 0 , Section 6]), we have the relation

$$
\mathbb{E}\left[\frac{\sinh B_{t} \sin \left(\frac{\pi}{2 t} B_{t}\right)}{\cosh B_{t}+\cosh x}\right]=\exp \left(-\frac{\pi^{2}}{8 t}\right) \mathbb{E}\left[\frac{1}{\cosh \left(x+B_{t}\right)}\right]
$$

for any $x \in \mathbb{R}$. By rewriting

$$
\frac{1}{\cosh \left(x+B_{t}\right)}=\frac{2\left(\cosh x \cosh B_{t}-\sinh x \sinh B_{t}\right)}{\cosh \left(2 B_{t}\right)+\cosh (2 x)}
$$

it also holds that

$$
\mathbb{E}\left[\frac{\sinh B_{t} \sin \left(\frac{\pi}{2 t} B_{t}\right)}{\cosh B_{t}+\cosh x}\right]=2 \exp \left(-\frac{\pi^{2}}{8 t}\right) \cosh x \mathbb{E}\left[\frac{\cosh B_{t}}{\cosh \left(2 B_{t}\right)+\cosh (2 x)}\right] .
$$

Finally, by dividing both sides of (A.10) by $\alpha \neq 0$ and letting $\alpha \rightarrow 0$, it follows that

$$
\frac{1}{t} \mathbb{E}\left[\frac{B_{t} \sinh B_{t}}{\cosh B_{t}+\cosh x}\right]=\mathbb{E}\left[\frac{1}{\cosh \left(x+B_{t}\right)+1}\right], \quad x \in \mathbb{R},
$$

because of the fact that $\pi \xi / \sinh (\pi \xi), \xi \in \mathbb{R}$, is the Fourier transform of the probability density $\{2(\cosh x+1)\}^{-1}, x \in \mathbb{R}$, which may be seen from (A.8) by letting $b \rightarrow 0$ on both sides (see also [25] as cited in the case $\alpha=\pi / 2$ above).

Acknowledgements. This work was partially supported by JSPS KAKENHI Grant Number 17K05288. 


\section{References}

[1] L. Alili, J.-C. Gruet, An explanation of a generalized Bougerol's identity in terms of hyperbolic Brownian motion, in: Exponential functionals and principal values related to Brownian motion, A collection of research papers, M. Yor (ed.), pp. 15-33, Biblioteca de la Revista Mathemática Iberoamericana, Rev. Mat. Iberoamericana, Madrid, 1997.

[2] T. Assiotis, A matrix Bougerol identity and the Hua-Pickrell measures, Electron. Commun. Probab. 23 (2018), no. 7, 1-11.

[3] O. Barndorff-Nielsen, Exponentially decreasing distributions for the logarithm of particle size, Proc. R. Soc. Lond. A 353 (1977), 401-419.

[4] O. Barndorff-Nielsen, Hyperbolic distributions and distributions on hyperbolae, Scand. J. Statist. 5 (1978), 151-157.

[5] O. Barndorff-Nielsen, P. BlÆsild, C. Halgreen, First hitting time models for the generalized inverse Gaussian distribution, Stoch. Process. Appl. 7 (1978), 49-54.

[6] A.N. Borodin, P. Salminen, Handbook of Brownian Motion - Facts and Formulae, corrected reprint of 2nd ed., 2002, Birkhäuser, Basel, 2015.

[7] Ph. Bougerol, Exemples de théorèmes locaux sur les groupes résolubles, Ann. Inst. H. Poincaré Sect. B (N.S.) 19 (1983), 369-391.

[8] A. Comtet, C. Monthus, M. Yor, Exponential functionals of Brownian motion and disordered systems, J. Appl. Probab. 35 (1998), 255-271, also in: [31, pp. 182-203.

[9] D. Dufresne, The distribution of a perpetuity, with applications to risk theory and pension funding, Scand. Actuar. J. 1990 (1990), 39-79.

[10] R. Durrett, Probability: Theory and Examples, 4th ed., Cambridge Univ. Press, Cambridge, 2010.

[11] H. Geman, Functionals of Brownian motion in finance and in insurance, in: [31], pp. $1-13$.

[12] I.S. Gradshteyn, I.M. Ryzhik, Table of Integrals, Series, and Products, 8th ed., Elsevier/Academic Press, Amsterdam, 2015.

[13] J. Jakubowski, M. Wiśniewolski, On hyperbolic Bessel processes and beyond, Bernoulli 19 (2013), no. 5B, 2437-2454.

[14] B. Jørgensen, Statistical Properties of the Generalized Inverse Gaussian Distribution, Lect. Notes in Statist. 9, Springer, New York, 1982. 
[15] I. Karatzas, S.E. Shreve, Brownian Motion and Stochastic Calculus, 2nd ed., Springer, New York, 1991.

[16] K. Kawazu, H. Tanaka, On the maximum of a diffusion process in a drifted Brownian environment, in: Séminaire de Probabilités, XXVII, pp. 78-85, Lect. Notes in Math. 1557, Springer, Berlin, 1993.

[17] N.N. Lebedev, Special Functions and their Applications, Dover, New York, 1972.

[18] H. Matsumoto, S. Taniguchi, Stochastic Analysis: Itô and Malliavin Calculus in Tandem, Cambridge Univ. Press, Cambridge, 2017.

[19] H. Matsumoto, M. Yor, On Bougerol and Dufresne's identities for exponential Brownian functionals, Proc. Japan Acad. Ser. A Math. Sci. 74 (1998), 152-155.

[20] H. Matsumoto, M. Yor, An analogue of Pitman's $2 M-X$ theorem for exponential Wiener functionals, Part I: A time-inversion approach, Nagoya Math. J. 159 (2000), $125-166$.

[21] H. Matsumoto, M. Yor, An analogue of Pitman's $2 M-X$ theorem for exponential Wiener functionals, Part II: The role of the generalized inverse Gaussian laws, Nagoya Math. J. 162 (2001), 65-86.

[22] H. Matsumoto, M. Yor, On Dufresne's relation between the probability laws of exponential functionals of Brownian motions with different drifts, Adv. in Appl. Probab. 35 (2003), 184-206.

[23] H. Matsumoto, M. Yor, Exponential functionals of Brownian motion, I: Probability laws at fixed time, Probab. Surv. 2 (2005), 312-347.

[24] H. Matsumoto, M. Yor, Exponential functionals of Brownian motion, II: Some related diffusion processes, Probab. Surv. 2 (2005), 348-384.

[25] D. Revuz, M. Yor, Continuous Martingales and Brownian Motion, 3rd ed., Springer, Berlin, 1999.

[26] V. Seshadri, The Inverse Gaussian Distribution: A Case Study in Exponential Families, Oxford Univ. Press, New York, 1993.

[27] S. Vakeroudis, Bougerol's identity in law and extensions, Probab. Surv. 9 (2012), 411-437.

[28] G.N. Watson, A Treatise on the Theory of Bessel Functions, 2nd ed., Cambridge Univ. Press, Cambridge, 1944.

[29] M. Yor, Loi de l'indice du lacet Brownien, et distribution de Hartman-Watson, Z. Wahrsch. Verw. Gebiete 53 (1980), 71-95. 
[30] M. Yor, On some exponential functionals of Brownian motion, Adv. in Appl. Probab. 24 (1992), 509-531, also in: [31], pp. 23-48.

[31] M. Yor, Exponential Functionals of Brownian Motion and Related Processes, Springer, Berlin, 2001. 